\title{
13.
}

\section{Recherches sur la résolution des équations de tous les degrés.}

\author{
(Par M. le colonel F. Theremin à Saratoff en Russie.)
}

\section{Contenu des articles.}

Art. I. Recherche d'une expression générale des racines d'une équation d'un degré quelconque, en fonction des coefficients de l'équation, et des racines d'une équation auxiliaire.

Art. II. Application à l'équation générale du second degré. - But qu'il faut se proposer.

Art. III. Déduction d'une expression générale des racines d'une équation d'un degré quelconque, en fonction d'une quantité entièrement arbitraire $\alpha$.

Art. IV. Parti que l'on peut tirer de cette expression générale, qui est une série. Approximation linéaire. - Déduction d'une nouvelle série plus simple.

Art. V. Examen général des formes et des propriétés des lieux géométriques des équations.

Art. VI. Propriétés correspondantes des équations et des lieux géométriques qu'elles représentent. - Démonstration géométrique de la règle des signes, employée par Fourrier pour la séparation des racines.

Art. VII. Choix de la quantité arbitraire; - détermination des racines, lorsque toutes celles de l'équation sont réelles. - Application à une équation du $3^{\text {ine }}$ degré. Observation sur le tracé des lieux gométériques.

Art. Vlil. Examen du cas des racines égales, et de celui des racines imaginaires ou manquantes. - Points singuliers simples et composés. Règle pour s'assurer si deux racines sont égales ou inégales ou imaginaires, quand le lieu géométrique de l'équation a un point de maximum ou de minimum, très près de l'axe des $x$.

Art. IX. Convergence de la formule. - Moyen de s'assurer du nombre de chiffres décimaux exacts, à chaque opération.

Art. X. Application à une équation du $7^{\text {me }}$ degré.

Art. XI. Emploi de la méthode pour l'extraction des racines d'un degré quelconque. Application. 
Essai sur la résolution des équations de tous les degrés.

\section{I.}

La résolution d'une équation peut s'entendre de deux manières:

$1^{\circ}$. Ou l'on se propose de trouver l'inconnue $x$ d'une manière générale en fonction des coefficients de l'équation; - ou,

$2^{\circ}$. On se propose de déterminer successivement chacune des racines de cette équation.

La détermination des racines est toujours le but final de la résolution d'une équation, parconséquent la recherche de l'expression générale de l'inconnue en fonction des coefficients, ne forme que la première partie du problême proposé, et la solution finale doit pouvoir se déduire de cette expression générale.

Examinons successivement ces deux parties du problème, et proposons nous d'abord, de trouver l'expression générale de l'inconnue $x$, d'une équation d'un degré quelconque $m$, en fonction des coefficients de cette équation.

L'équation générale du degré $m$ est:

$$
\boldsymbol{x}^{m}+\boldsymbol{A} \boldsymbol{x}^{m-1}+\boldsymbol{B} \boldsymbol{x}^{m-2}+\cdots \cdot \boldsymbol{K} x^{2}+\boldsymbol{L} \boldsymbol{x}+\boldsymbol{M}=\mathbf{0}
$$

dans laquelle $\dot{m}$ est un nombre entier positif, et où $\boldsymbol{A}, \boldsymbol{B}, \boldsymbol{C}$, etc. $\boldsymbol{M}$ sont des nombres réels, entiers ou fractionnaires, positifs ou négatifs.

Si l'on suppose pour un moment que la quantité $\boldsymbol{M}$ devienne variable et qu'on la désigne par $-y$, en la faisant passer dans le second membre, on aura:

$$
\boldsymbol{x}^{m}+\boldsymbol{A} \boldsymbol{x}^{m-1}+\boldsymbol{B} \boldsymbol{x}^{m-2}+\cdots \cdot \boldsymbol{K} \boldsymbol{x}^{2}+\boldsymbol{L} \boldsymbol{x}=\boldsymbol{y}
$$

équation qui correspond à la proposée dans le cas où la variable $y$ devient égal à $-\boldsymbol{M}$.

Le problême se trouve donc ramené à trouver l'expression de la variable $x$ en fonction de la variable $y$, expression que nous désignerons d'une manière générale par:

$$
x=f(y) \text {. }
$$

Si nous désignons par: $f^{\prime}(y), f^{\prime \prime}(y)$, etc. les dérivées successives de $f(y)$, et par: $f(y)_{0}, f^{\prime}(y)_{0}, f^{\prime \prime}(y)_{0}$, etc. ce que deviennent cette fonction 
et ses dérivées quand on $\mathrm{y}$ fait $y=0$, nous aurons en général par la série de Maclaurin, l'expression

$$
x=f(y)_{0}+\frac{f^{\prime}(y)_{0}}{1} y+\frac{f^{\prime \prime}(y)_{0}}{1.2} y^{2}+\frac{f^{\prime \prime \prime}(y)_{0}}{1.2 .3} y^{3}+\text { etc. },
$$

dans laquelle il s'agit de déterminer les termes $f(y)_{0}, f^{\prime}(y)_{0}$; etc.

Pour cela reprenons l'équation générale:

$$
\boldsymbol{x}^{m}+\boldsymbol{A} \boldsymbol{x}^{m-1}+\boldsymbol{B} \boldsymbol{x}^{m-2}+\cdots \boldsymbol{K} x^{2}+\boldsymbol{L} \boldsymbol{x}=y,
$$

nous aurons en différentiant:

$$
\left(m x^{m-1}+(m-1) A x^{m-2}+\cdots \cdot 2 K x+L\right) d x=d y,
$$

d'où l'on tire:

$$
\frac{d x}{d y}=\frac{1}{m x^{m-1}+(m-1) A x^{m-2}+\cdots \cdots 2 K x+L},
$$

et parconséquent:

$$
f^{\prime}(y)=\frac{1}{m x^{m-1}+(m-1) A x^{m-2}+\cdots \cdots 2 K x+L} .
$$

Si l'on fait pour abréger:

$$
\boldsymbol{X}=\boldsymbol{x}^{\boldsymbol{m}}+\boldsymbol{A} \boldsymbol{x}^{m-1}+\cdots \boldsymbol{L} \boldsymbol{x}+\boldsymbol{m},
$$

et que l'on désigne par $\boldsymbol{X}^{\prime}, \boldsymbol{X}^{\prime \prime}$, etc. les dérivées successives de ce polynome, il est évident que l'expression ci-dessus deviendra:

$$
\frac{d x}{d y}=f^{\prime}(y)=\frac{1}{X^{\prime}}
$$

et que par la différentiation on en tirera successivement

$$
\begin{aligned}
& \frac{d^{2} x}{d y^{2}}=f^{\prime \prime}(y)=-\frac{X^{\prime \prime}}{\overline{\bar{X}^{3}}}, \\
& \frac{d^{3} x}{d y^{3}}=f^{\prime \prime \prime}(y)=+\frac{\left(3 \overline{X^{\prime \prime}}-X^{\prime} X^{\prime \prime \prime}\right)}{\overline{X^{\prime}}},
\end{aligned}
$$

Pour savoir maintenant ce que deviennent $f(y)$ et ses dérivées successives quand on $\mathrm{y}$ fait $y=0$, reprenons l'équation générale en $x$ et en $y$, et posons y $y=0$, nous aurons:

$$
\text { (1.) } \boldsymbol{x}^{m}+\boldsymbol{A} \boldsymbol{x}^{m-1}+\cdots \cdots \boldsymbol{K} \boldsymbol{x}^{2}+\boldsymbol{L} \boldsymbol{x}=\mathbf{0} \text {, }
$$

et, comme $x$ est facteur commun, nous aurons séparément:

et :

$$
\boldsymbol{x}=\mathbf{0} \text {, }
$$

$$
\boldsymbol{x}^{m-1}+\boldsymbol{A} \boldsymbol{x}^{m-2}+\cdots \boldsymbol{K} \boldsymbol{x}+\boldsymbol{L}=\mathbf{0}
$$


La première de ces équalions donne:

$$
x=f(y)_{0}=0
$$

et réduit les quantités $\boldsymbol{X}^{\prime}, \boldsymbol{X}^{\prime \prime}, \boldsymbol{X}^{\prime \prime \prime}$, etc. au terme indépendant de $x$.

La seconde équation est, comme on voit, d'un degré moins élevé de l'unité, que celư de l'équation donnée, et il est évident qu'en nommant $\alpha, \beta, \gamma$, etc. les $i n-1$ racines de cette équation, il faudra successivement substituer ces racines au lieu de $x$, dans les polynomes $\boldsymbol{X}^{\prime}, \boldsymbol{X}^{\prime \prime}, \boldsymbol{X}^{\prime \prime \prime}$, etc. et nous obtiendrons par là autant de valeurs correspondantes des quantités: $f^{\prime}(y), f^{\prime \prime}(y), 0$, etc.

De cette manière, on voit que la résolution de l'équation générale du degré $m$, dépend de la résolution d'une autre équation, qui est aussi du degré $m$, mais dont l'une des racines est constamment égale à zéro, et qui se réduit parconséquent á une équation d'un degré $m-1$; - nous nommerons uuxiliaire l'équation (1.) dont dépend la résolution de l'équation donnée.

Chacune des racines de l'équation auxiliaire réduit à zéro la fonction $y$, et si nous désignons en général l'une quelconque de ces racines par $r$, et par

$$
\boldsymbol{X}_{(r)}, \quad \boldsymbol{X}_{(r)}^{\prime}, \quad \boldsymbol{X}_{(r)}^{\prime \prime}, \text { etc., }
$$

ce que deviennent $\boldsymbol{X}$ et ses dérivées quant on y substitue $r$ au lieu de $x$, alors l'expression générale de $x$ en $y$ deviendra:

$$
x=r+\frac{1}{X_{(r)}^{\prime}} \cdot \frac{y}{1}-\frac{X_{(r)}^{\prime \prime}}{{\overline{X_{(r)}^{\prime}}}_{(3)}^{\prime}} \cdot \frac{y^{2}}{1.2}+\frac{\left(3{\overline{X_{(r)}^{\prime \prime}}}^{\prime 2}-X_{(r)}^{\prime} X_{(r)}^{\prime \prime \prime}\right)}{{\overline{X_{(r)}^{\prime}}}^{5}} \frac{y^{3}}{1.2 .3}+\text { etc. }
$$

et, comme dans le cas de l'équation donnée la variable $y$ devient égale à $-M$, on aura:

$$
\text { (2.) } \quad x=r-\frac{1}{X_{(r)}^{\prime}} \cdot \frac{M}{1}-\frac{X_{(r)}^{\prime \prime}}{{\overline{X_{(r)}^{3}}}_{(3)}} \cdot \frac{M^{2}}{1.2}-\frac{\left(3{\overline{X_{(r)}^{\prime \prime}}}^{2}-X_{(r)}^{\prime} X_{(r))}^{\prime \prime \prime}\right.}{{\overline{X^{\prime}}}^{5}} \frac{M^{3}}{1.2 .3}+\text { etc. }
$$

pour l'expression générale de toutes les racines de l'équation du degré $m$.

Il est bon d'observer que l'une des racines $(\boldsymbol{r})$ de l'équation auxiliaire étant toujours égale à zéro, on a directement l'une des racines de la proposée en faisant: $r=0$ dans l'expression (2.); - les $m-1$ racines restantes, dépendront des $m-1$ valeurs de $r$ autres que zéro, et qui sont les racines d'une équation d'un degré $m-1$.

On voit que l'expression de $x$ est de la plus grande généralité possible, puisqu'elle est indépendante de toute hypothèse, non seulement sur la valeur des coefficients, mais même sur, le degré de l'équation proposée. - Sans doute, vu la généralité du problême, on ne pouvait pas s'attendre à une expression, 
soit finie, soit plus explicite, mais on peut dès à présent considérer comme résolue la première partie du problême, sauf à ramener l'expression générale (2.) soit à une formule finie, soit à une série convergente et d'une application facile à la détermination numérique des racines.

\section{II.}

Avant de pousser plus loin ces recherches, et comme vérification de la méthode proposée, faisons l'application de l'expression générale (2.), à la détermination de l'inconnue dans l'équation générale du second degré:

$$
\boldsymbol{x}^{2}+\boldsymbol{A} \boldsymbol{x}+\boldsymbol{B}=\mathbf{0} .
$$

Nous voyons d'abord que les polynomes $\boldsymbol{X}, \boldsymbol{X}^{\prime}, \boldsymbol{X}$, etc. deviennent dans ce cas particulier:

$$
\begin{aligned}
& \boldsymbol{X}=x^{2}+\boldsymbol{A} x+\boldsymbol{B} \\
& \boldsymbol{X}^{\prime}=2 x+\boldsymbol{A}, \\
& \boldsymbol{X}^{\prime \prime}=2, \\
& \boldsymbol{X}^{\prime \prime \prime}=0, \text { aussi bien que toutes les dérivées suivantes. }
\end{aligned}
$$

De plus, l'équation auxiliaire est ici

$$
x^{2}+A x=0
$$

qui donne: $x=0$, et $x+\boldsymbol{A}=0$, d'où l'on tire $x=-\boldsymbol{A}$.

En mettant ces deux valeurs de $x$ dans $X^{\prime}$ et $X^{\prime \prime}$, on aura:

$$
\begin{aligned}
& \text { pour } x=0 \text { et pour } x=-A \text {, } \\
& \boldsymbol{X}^{\prime}=+\boldsymbol{A} \quad \boldsymbol{X}^{\prime}=-\boldsymbol{A}, \\
& X^{\prime \prime}=+2 \quad X^{\prime \prime}=+2 \text {. }
\end{aligned}
$$

En mettant ces valeurs dans l'expression générale (2.), on aura:

$$
\begin{gathered}
\text { pour } x=0 \\
x=0+\frac{1}{A} \cdot \frac{y}{1}-\frac{2}{A^{3}} \cdot \frac{y^{2}}{1.2}+\frac{3 .(2)^{2}}{A^{5}} \cdot \frac{y^{3}}{1.2 .3}-\frac{3.5 \cdot(2)^{3}}{A^{7}} \cdot \frac{y^{4}}{1.2 .3 .4}+\text { etc. } \\
\text { et pour } x=-A \\
x=-A-\frac{1}{A} \cdot \frac{y}{1}+\frac{2}{A^{3}} \cdot \frac{y^{2}}{1.2}-\frac{3 .(2)^{2}}{A^{5}} \cdot \frac{y^{3}}{1.2 .3}+\frac{3.5 \cdot(2)^{3}}{A^{7}} \cdot \frac{y^{4}}{1.2 .3 .4}-\text { etc. },
\end{gathered}
$$

Mais en observant que le premier terme de la première de ces expressions, qui est zéro, peut être remplacé par: $-\frac{1}{2} \boldsymbol{A}+\frac{1}{2} \boldsymbol{A}$, et que le premier terme $-\boldsymbol{A}$ de la seconde peut être remplacé par $-\frac{1}{2} \boldsymbol{A}-\frac{1}{2} \boldsymbol{A}$, on verra qu'en faisant cette substitution, le premier terme de chacune des deux séries sera $-\frac{1}{2} \boldsymbol{A}$, et que tous les termes suivants dans les deux séries sont égaux 
un à un mais de signes contraires, parconséquent les deux valeurs de $x$ pourront être réunies en une seule expression qui sera:

$$
x=-\frac{1}{2} A \pm\left\{\frac{1}{2} A+\frac{1}{A} \cdot \frac{y}{1}-\frac{2}{A^{3}} \cdot \frac{y^{2}}{1.2}+\frac{3 .(2)^{2}}{A^{5}} \cdot \frac{y^{8}}{1.2 .3}-\cdots \cdots\right\},
$$

et qui deviendra l'expression des racines de l'équation donnée, quand on $\mathrm{y}$ fera $y=-B$; - faisant cette substitution et réduisant, il vient:

$$
x=-\frac{1}{2} A \pm\left\{\frac{1}{2} A-\frac{B}{A}-\frac{B^{2}}{A^{3}}-\frac{2 B^{3}}{A^{5}}-\frac{5 B^{4}}{A^{7}}-\cdots \cdot\right\} \text {. }
$$

Mais avec un peu d'attention on voit que la quantité comprise entre parenthèses n'est autre chose que le développement en série du radical:

$$
\sqrt{ }\left(\frac{1}{4} A^{2}-B\right) \text { ou } \sqrt{1} \frac{1}{4}\left(A^{2}-4 B\right),
$$

parconséquent l'expression trouvée se réduit à:

$$
x=-\frac{1}{2} A \pm \frac{1}{2} \sqrt{ }\left(A^{2}-4 B\right)
$$

qui est l'expression connue des racines de l'équation du second degré.

Au moyen du développement (2.) on pourrait facilement déduire les séries générales qui exprimeraient les racines de l'équation du $3^{\text {me }}$ degré, puis de celles du $4^{\text {ine }}$ du $5^{\text {me }}$, et ainsi de suite, comme nous l'avons fait pour l'équation du $2^{\text {d }}$ degré; mais, pour que ces séries puissent servir à la détermination numérique des racines, il faut nécessairement que l'on parvienne à remplir l'une des deux conditions suivantes:

$1^{\circ}$. Il faut que les séries soyent, ou puissent être rendus convergentes; - ou bien:

$z^{\circ}$. Il faut qu'elles puissent se transformer, comme pour le second degré, en formules finies, algèbriques, qui donneraient les racines de la proposée par un nambre fini d'opérations arithmétiques.

Mais on comprendra facilement, que l'opération de ramener à une expression finie les développements des racines des équations, d'un degré supérieur au second, ne serait tout au plus possible que pour les équations du $3^{\text {me }}$ et peut être pour celles du $4^{\text {ine }}$ degré (ce qui est même fort douteux), mais que cette transformation deviendrait entièrement impossible pour les degrés supérieurs. - D'ailleurs, en admettant même la possibilité de cette transformation pour tous les degrés de l'échelle infinie des nombres, il faudrait déduire autant de formules différentes qu'il y a de nombres, c'est à dire une infinité, et parconséquent la question ne serait jamais résolue. 
De même, si l'on cherche à rendre convergent le développement des racines d'une équation d'un degré déterminé, par exemple du $3^{\text {me }}$, il faudra de nouvelles recherches pour le $4^{\text {me }}$, puis pour le cinquième, et ainsi de suite à l'infini.

Il est donc inutile de s'occuper de recherches semblables, et les efforts de l'analyse doivent tendre: soit à rendre convergente l'expression générale (2.), soit à découvrir une autre série convergente, indépendemment de toute hypothèse sur le degré de l'équation. - C'est ce que je vais tâcher de faire dans les articles suivants.

\section{III.}

L'expression (2.) donne la racine de l'équation au moyen d'une suite infinie de termes, formés des coefficients de l'équation et de l'une des racines de l'équation auxiliaire; chacun des termes à partir du second est multiplié par l'une des puissances successives de $\boldsymbol{M}$, et divisé par l'une des puissances successives impaires de $\boldsymbol{X}_{(r)}^{\prime}$, de manière que le second terme a pour facteur: $\frac{M}{X_{(r)}^{\prime}}$, le troisième: $\frac{M^{2}}{\bar{X}_{(r)}^{3}}$, le quatrième: $\frac{M^{3}}{{\overline{X^{\prime}}}_{(r)}^{5}}$, etc., d'où l'on voit que l'une des premières conditions de la convergence serait que: $X_{(r)}^{\prime}$ fût plus grand que $M$, abstraction faite du signe de ces quantités; - or, cette condition ne saurait évidemment être remplie qu'accidentellement, puisque $\boldsymbol{M}$ est un nombre donné qu'ón ne saurait changer, et que $\boldsymbol{X}_{(r)}^{\prime}$ est une quantité qui dépend de la racine $\boldsymbol{r}$ de l'équation auxiliaire, supposée connue. - Mais nous allons voir qu'on peut facilement déduire un autre série de forme semblable, et qui jouit de la propriété de pouvoir être rendue aussi convergente qu'on voudra.

Pour cela reprenons l'équation donnée, et supposons que le second membre de celte équation au lieu d'être zéro, soit une variable $y$, nous aurons:

$$
\boldsymbol{x}^{m}+\boldsymbol{A} \boldsymbol{x}^{m-1}+\boldsymbol{B} \boldsymbol{x}^{m-2}+\cdots \cdot \boldsymbol{K} \boldsymbol{x}^{2}+\boldsymbol{L} \boldsymbol{x}+\boldsymbol{M}=\boldsymbol{y} .
$$

Cette équation est celle d'une courbe dont $x$ est l'abscisse et $y$ l'ordonnée, et les racines de l'équation proposée correspondront au cas de $y=0$, c'est à dire seront les abscisses des points où la courbe coupe ou touche l'axe des abscisses.

Soit $\alpha$ une quantité arbitraire quelconque prise pour abscisse de la courbe, et $\delta$ l'ordonnée correspondante, on aura:

$$
\alpha^{m}+\boldsymbol{A} \alpha^{m-1}+\boldsymbol{B} \alpha^{m-2}+\cdots \cdot+\boldsymbol{K} \alpha^{2}+\boldsymbol{L} \alpha+\boldsymbol{M}=\delta
$$


en retranchant cette équation de l'équation (3.), on aura:

$$
\begin{gathered}
\text { (4.) } \quad \boldsymbol{x}^{m}+\boldsymbol{A} \boldsymbol{x}^{m-1}+\cdots \cdots+\boldsymbol{K} \boldsymbol{x}^{2}+\boldsymbol{L} \boldsymbol{x}+\boldsymbol{M} \\
-\left(\alpha^{m}+\boldsymbol{A} \alpha^{m-1}+\cdots \cdots \boldsymbol{K} \dot{\alpha}+\boldsymbol{L} \alpha+\boldsymbol{M}\right)=y-\delta .
\end{gathered}
$$

Si nous posons: $y-\delta=z$, nous pourrons considérer $z$ comme une nouvelle variable, tandis que $\alpha$ conservera une valeur constante, et nous aurons d'après la série de Maclaurin:

$$
x=f(z)_{0}+f^{\prime}(z)_{0} \frac{z}{1}+f^{\prime \prime}(z)_{0} \frac{z^{2}}{1.2}+f^{\prime \prime \prime}(z)_{0} \frac{z^{3}}{1.2 .3}+\cdots
$$

développement dans lequel il s'agit de déterminer la fonction $f(z)$ et ses derivées dans le cas où l'on $y$ fait $z=0$.

Si l'on différentie l'équation (4.) en mettant dans le second membre $z$ au lieu de $y-\delta$, on aura:

ou bien

$$
m x^{m-1}+(m-1) A x^{m-2}+\cdots \cdot 2 K x+L=\frac{d z}{d x},
$$

$$
\dot{X}^{\prime}=\frac{d z}{d x}, \text { d'où l'on tire: } \frac{d x}{d z}=f^{\prime}(z)=\frac{1}{X^{\prime}},
$$

et successivement:

$$
f^{\prime \prime}(z)=-\frac{X^{\prime \prime}}{\overline{X^{\prime}}}, \quad f^{\prime \prime \prime}(z)=+\frac{\left(3 \overline{X^{\prime \prime}}-X^{\prime} X^{\prime \prime \prime}\right)}{{\overline{X^{\prime}}}^{5}}, \ldots
$$

Pour savoir ce que deviennent ces dérivées et la fonction primitive $f(z)$ quand on $y$ fait $z=0$, il faut égaler à zéro le second membre de l'équation (4.) d'où l'on tire:

et

$$
y=\delta
$$

$$
\boldsymbol{x}^{m}+\boldsymbol{A} \boldsymbol{x}^{m-1}+\cdots \cdot \boldsymbol{L} \boldsymbol{x}+\boldsymbol{M}-\left(\alpha^{m}+\boldsymbol{A} \boldsymbol{\alpha}^{m-1}+\cdots \cdot \boldsymbol{L} \alpha+\boldsymbol{M}\right)=\mathbf{0}
$$

Cette dernière équation peut se mettre sous la forme:

$$
\text { (5.) } \quad x^{m}-\alpha^{m}+\boldsymbol{A}\left(x^{m-1}-\alpha^{m-1}\right)+\cdots \cdot+\boldsymbol{L}(x-\alpha)=0,
$$

cette équation étant évidemment divisible par $x-\alpha$, il s'ensuit que nous aurons

$$
x=\alpha \text {, pour l'une des valeurs de: } x=f(z)_{10} \text {, }
$$

et les autres $m-1$ valeurs sẹront les $m-1$ racines de l'équation qui résultera de la division de l'équation (5.) par: $x-\alpha$.

L'équation (5.) est évidemment l'équation auxiliaire de la proposée, il $y$ a seulement cette différence entre l'équation (1.) et celle-ci, que dans la première, l'une des racines est constamment égale à zéro, tandis que dans 
la dernière l'une des pacines est constamment égale à la quantité arbitraire $\alpha$. Nous verrons que l'introduction de cetle quantité arbitraire dispense de la recherche des $m-1$ racines restantes de l'équation auxiliaire.

Mettant donc $\alpha$ au lieu de $\boldsymbol{f}(\boldsymbol{z})_{0}$, et désignant par $\boldsymbol{X}_{(\alpha)}^{\prime}, \boldsymbol{X}_{(\alpha)}^{\prime \prime}$, etc. les dérivées du polynome $\boldsymbol{X}$ quand on $\mathbf{y}$ fait $\boldsymbol{x}=\alpha$, nous aurons:

$$
x=\alpha+\frac{1}{X_{(\alpha)}^{\prime}} \cdot \frac{z}{1}-\frac{X_{(\alpha)}^{\prime \prime}}{{\overline{X_{(\alpha)}^{\prime}}}_{(\alpha)}} \cdot \frac{z^{2}}{2.3}+\frac{\left(3 \overline{X_{(\alpha)}^{\prime \prime}}-X_{(\alpha)}^{\prime} X_{(\alpha))}^{\prime \prime \prime}\right.}{{\overline{X_{(\alpha)}^{\prime}}}_{1}^{\prime}} \frac{z^{3}}{1.2 .3}-\cdots
$$

pour l'expression de $x$ en fonction de la variable $z$ et de la quantité arbitraire $\alpha$; - mais, comme $x$ devient une des racines de la proposée chaque fois que l'ordonnée $y$ devient zéro, il s'ensuit que pour avoir l'expression de la racine il faut faire $y=0$ dans l'équation $z=y-\delta$, ou substituer - $\delta$ à $z$ dans le développement ci-dessus; - enfin, comme $\delta$ n'est autre chose que l'ordonnée de la courbe au point dont l'abscisse est $\alpha$, et qu'on obtient la valeur de cette ordonnée en mettant $\alpha$ au lieu de $x$ dans le polynome $\boldsymbol{X}$, on voit que $\boldsymbol{z}$ doit être remplacé par $-\boldsymbol{X}_{(\alpha)}$, et l'on aura enfin:

$$
x=\alpha-\frac{X_{(\alpha)}}{X_{(\alpha)}^{\prime}}-\frac{1}{2} \frac{\bar{X}_{(\alpha)}^{2} X_{(\alpha)}^{\prime \prime}}{\bar{X}_{(\alpha)}^{3}}-\frac{1}{2.3} \frac{\bar{X}_{(\alpha)}^{3}\left(3{\overline{X_{(\alpha)}^{\prime \prime}}}^{2}-X_{(\alpha)}^{\prime} X_{(\alpha)}^{\prime \prime \prime}\right)}{{\overline{X_{(\alpha)}^{\prime}}}^{5}}-\cdots
$$

pour l'expression générale de l'une des racines d'une équation d'un degré quẹlconque $m$, en fonction d'une quantité entièrement arbitraire $\alpha$.

Le développement (2.) précédemment trouvé, avait tous ses termes déterminés par les racines de l'équation auxiliaire (1.); - dans le nouveau développement (6.) la quantité $\alpha$ dont les termes dépendent, étant arbitraire, représente non seulement toutes les racines de l'équation auxiliaire, mais tel nombre qu'on voudra, et peut parconséquent être choisie à volonté de la manière la plus convenable au but que l'on se propose, et, nous verrons dans les articles suivants, que c'est du choix de cette quantité que dépend la rapidité du calcul des racines de l'équation proposée.

\section{IV.}

Voyons le parti qu'on peut tirer du développement (6.), pour obtenir la racine de l'équation avec tel degré d'approximation qu'on voudra.

Pour cela, considérons une portion $C I C$ fig. $1(A)$ de la courbe qui représente l'équation donnée, vers l'un des points, $I$, où cette courbe coupe l'axe des $x$, c'est à dire vers un des points pour lesquels la variable $\boldsymbol{x}$ devient une des racines $\boldsymbol{O I}$ de l'équation proposée, et supposons que la

Crelle's Journal f. d. M. Bd. XLIX. Heft 3. 
quantité $\alpha$ qui est arbitraire, et qui forme le premier terme de la série (6.), soit moindre que l'abscissé $O I$, ou que la racine, et égale à une abscisse $O m$; supposons de plus qu'entre $n$ et $\boldsymbol{I}$ la courbe ne présente aucun point singulier.

Si l'on élève une perpendiculaire à l'axe des $x$, au point $m$, extrèmilé de l'abscisse $\alpha$; la partie $m n$ de cette perpendiculaire interceptée par l'axe et la courbe, sera l'ordonnée de cette dernière et son expression analytique est $\boldsymbol{X}_{(\alpha)}$; - ensuite, si par le point $\boldsymbol{n}$ on mène à la courbe la touchante $\| t^{\prime}$, cette touchante coupera l'axe des $x$ en un point $t^{\prime}$, et la distance $m t^{\prime}$ sera la soustangente de la courbe pour le point dont $\alpha$ est l'abscisse; - mais, puisque $\boldsymbol{X}_{(\alpha)}^{\prime}$ représente la tangente trigonométrique de l'angle que la touchante $\|^{\prime}$ fait avec l'axe des $x$, l'expression de la soustangente sera $\frac{X_{(\alpha)}}{X_{(\alpha)}^{\prime}}$, ce qui est justement le second terme du développement (6.) déduit à l'article précédent.

Il faut observer que dans la position de la courbe indiquée dans la fig. $1(\boldsymbol{A})$ l'ordonnée $\boldsymbol{X}_{(\alpha)}$ est positive, et la quantité $\boldsymbol{X}_{(\alpha)}^{\prime}$ est négative, c'est pourquoi le second terme de la série (6.) dans ce cas particulier devra prendre le signe $t$, de manière que, comme on le voit dans la figure, la première partie de l'approximation de la racine cherchée $O I$, sera $O m+m t^{\prime}$ ou $\alpha+\frac{X_{(\alpha)}}{X_{(\alpha)}^{\prime}}$, c'est à dire la somme de la quantité arbitraire $\alpha$ et de la soustangente.

Si la courbe était située de la manière qu'on voit dans $(B)$ fig. 1, alors l'ordonnée $m n$ ou $\boldsymbol{X}_{(\alpha)}$ serait négative, et la quantité $\boldsymbol{X}_{(\alpha)}^{\prime}$ serait au contraire positive, parconséquent dans ce cas là, le second terme de la série (6.) prendra encore le signe positif.

Si la courbe, au lieu de tourner sa convexité vers l'origine des coordonnées comme en $(\boldsymbol{A})$ et $(\boldsymbol{B})$, tournait au contraire sa concavité vers ce point, comme on le voit en $(C)$ et en $(D)$ fig. 1 , alors le terme $\frac{X_{(\alpha)}}{X_{(\alpha)}^{\prime}}$ serait aussi positif, puisque l'ordonnée et la tangente trigonométrique de l'angle que la touchante avec l'axe des $x$, sont de signes contraires, mais la soustangente dépasserait le point d'intersection $I$ de la courbe avec l'axe, et parconséquent: la somme des deux premiers termes du développement, surpassera la racine, tandis que dans les deux cas examinés précédemment, la somme des deux premiers termes était moindre que la racine. 
Dans les quatre cas que nous venons d'examiner, l'abscisse om ou $\alpha$ était supposée moindre que la racine cherchée. Si au contraire cette abscisse était plus grande comme en $\left(A^{\prime}\right),\left(B^{\prime}\right),\left(C^{\prime}\right)$ et $\left(D^{\prime}\right)$ (fig. 1) il est facile de voir que pour les cas $\left(\boldsymbol{A}^{\prime}\right)$ et $\left(\boldsymbol{B}^{\prime}\right)$ la première approximation donnerait pour la somme des deux $1^{\text {ers }}$ termes du développement une quantité $O \boldsymbol{n}-m \boldsymbol{t}^{\prime}=\boldsymbol{O} \boldsymbol{t}^{\prime}$, moindre que la racine $O I$, tandis que dans les cas $\left(C^{\prime}\right)$ et $\left(D^{\prime}\right)$ on aurait $\boldsymbol{O} m-m t^{\prime}=\boldsymbol{O} t^{\prime}$ plus grand que la racine cherchée.

Dans l'un comme dans l'autre cas, le reste du développement exprime la différence entre la racine cherchée, et la somme algébrique des deux premiers termes de ce développement.

On voit, que si la quantité $\alpha$ dont on est parti n'est pas beaucoup différente de la racine, la somme algébrique des deux premiers termes de la série donnera toujours une valeur qui différera encore moins de la racine que la quantité $\alpha$ elle même; - d'après cela, au lieu de chercher à construire ou à calculer les termes suivants de la série, il est beaucoup plus simple, de prendre la somme algébrique des deux premiers termes: $\alpha \pm \frac{X_{(\alpha)}}{X_{(\alpha)}^{\prime}}$ pour une nouvelle quantité arbitraire que nous nommerons $\alpha^{\prime}$, et qui mise dans l'expression (6.) donne:

$$
x=\alpha^{\prime}-\frac{X_{\left(\alpha^{\prime}\right)}}{X_{\left(\alpha^{\prime}\right)}^{\prime}}-\frac{X_{\left(\alpha^{\prime}\right)}^{2} X_{\left(\alpha^{\prime}\right)}^{\prime \prime}}{2 \cdot \overline{\boldsymbol{X}}_{\left(\alpha^{\prime}\right)}^{\prime 3}}-\cdots
$$

Mais, en nous arrêtant au second terme, et posant:

$$
\alpha^{\prime}-\frac{X_{\left(\alpha^{\prime}\right)}}{X_{\left(\alpha^{\prime}\right)}^{\prime}}=\alpha^{\prime \prime}
$$

nous pourrons former une nouvelle série en prenant pour quantité arbitraire la quantité $\alpha^{\prime \prime}$ plus approchée de la racine que $\alpha^{\prime}$, et nous aurons:

$$
x=\alpha^{\prime \prime}-\frac{X_{\left(\alpha^{\prime \prime}\right)}}{\bar{X}_{\left(\alpha^{\prime \prime}\right)}^{\prime}}-\frac{X_{\left(\alpha^{\prime \prime}\right)}^{2} X_{\left(\alpha^{\prime \prime}\right)}^{\prime \prime}}{2 \cdot \bar{X}_{\left(\alpha^{\prime \prime}\right)}^{3}}-\cdots \cdots
$$

et ainsi de suite; - enfin remplaçant $\alpha^{\prime \prime}$ par sa valeur en $\alpha^{\prime}$, et $\alpha^{\prime}$ par sa valeur en $\alpha$, nous aurons le nouveau développement:

$$
\text { (7.) } \quad x=\alpha-\frac{X_{(\alpha)}}{X_{(\alpha)}^{\prime}}-\frac{X_{\left(\alpha^{\prime}\right)}}{X_{\left(\alpha^{\prime}\right)}^{\prime}}-\frac{X_{\left(\alpha^{\prime \prime}\right)}}{X_{\left(\alpha^{\prime \prime}\right)}^{\prime}}-\frac{\boldsymbol{X}_{\left(\alpha^{\prime \prime \prime}\right)}}{\boldsymbol{X}_{\left(\alpha^{\prime \prime \prime}\right)}^{\prime}}-\cdots
$$

dont la convergence est évidente, puisque les termes $\frac{\boldsymbol{X}_{(\alpha)}}{X_{(a)}^{\prime}}, \frac{X_{\left(\alpha^{\prime}\right)}}{X_{\left(\alpha^{\prime}\right)}^{\prime}}, \ldots$ ne sont autre chose que les soustangentes de la courbe, en des points, dont les abscisses ont ces soustangentes pour différences. 
Il faut ici faire deux observations importantes.

$1^{\circ}$. Quand l'extrêmité de l'abscisse $\alpha$ se trouve située du côté vers lequel la courbe tourne sa concavité, alors, la première approximation fait passer la quantité $\alpha^{\prime}$ qui en résulte, de l'autre côté du point d'intersection de la courbe avec l'axe des abscisses, et fait parconséquent rentrer ce cas, dès la première approximatión, dans celui où le point de départ est du côté de la convexité; et, pour ce dernier cas, il est évident que chaque nouvelle approximation s'approchera de la racine, sans la dépasser jamais, soit que $\alpha$ soit plus grand, soit qu'il soit moindre que la racine.

$2^{\circ}$. Quand le point de départ, ou l'extrêmité de l'abscisse $\alpha$, est situé du côté de la convexité de la courbe, quelque grande que soit la différence entre la racine, et $\alpha$, s'il ne se trouve d'ailleurs aucun point singulier sur la portion de courbe correspondante à ces deux points, on approchera de la racine assez rapidement, et chaque nouvelle valeur approchée $\alpha^{(n)}$ sera comprise entre la valeur approchée précédente $\alpha^{(n-1)}$, et la racine.

Si entre l'extrêmité de l'abscisse arbitraire $\alpha$, et la racine, la portion correspondante de la courbe présentait un point singulier, il pourrait arriver que la quantité $\boldsymbol{X}_{(\alpha)}^{\prime}$, ou l'une des suivantes $\boldsymbol{X}_{\left(\alpha^{\prime}\right)}^{\prime}, \boldsymbol{X}_{\left(\alpha^{\prime \prime}\right)}^{\prime}$, etc. devienne zéro, ce qui rendait le résultat infini, ét parconséquent ne ferait pas connaître la racine. - On voit donc, qu'avant d'aller plus loin, et pour être guidé dans le choix de la quantité arbitraire $\alpha$, qui doit servir de point de départ au calcul de chaque racine, il est indispensable d'étudier les formes et les propriétés de la courbe qui correspond à une équation d'un degré quelconque $m$, et les phénomenes de courbure qu'elle peut présenter. - C'est ce que nous allons faire dans les articles suivants.

\section{V.}

Commençons donc par examiner les propriétés et les formes générales des courbes qui correspondent à l'équation générale :

$$
\boldsymbol{X}=\boldsymbol{x}^{\boldsymbol{n}^{n}}+\boldsymbol{A} \boldsymbol{x}^{m-1}+\boldsymbol{B} \boldsymbol{x}^{m-2}+\cdots \cdot \boldsymbol{K} \boldsymbol{x}^{2}+\boldsymbol{L} \boldsymbol{x}+\boldsymbol{M}=\boldsymbol{y} .
$$

$1^{\circ}$. Nous -voyons d'abord que si l'on fait croître l'abscisse $x$ par degrés insensibles depuis $-\frac{1}{0}$ jusqu'à $+\frac{1}{0}$, les valeurs correspondantes de $y$ seront toutes réelles, et parconséquent la courbe est continue depuis $x=-\frac{1}{d}$ jusqu'à $x=+\frac{1}{0}$.

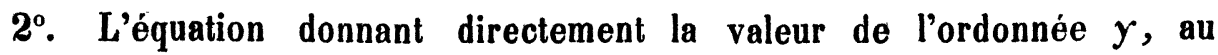
$1^{\text {er }}$ degré, il ne saurait parconséquent $y$ avoir plus d'une valeur de $y$, pour 
une seule et même valeur de $x$; - ainsi, la courbe n'aura pas de parties rentrantes dans le sens des $x$, c'est à dire telles que $x$ diminue après avoir augmenté, car si cela était, il y aurait des ordonnées qui couperaient la courbe en deux points, ou plus, et alors $y$ aurait plus d'une valeur correspondante à la même abscisse, ce qui ne peut être.

$3^{\circ}$. La courbe en aucun de ses points ne peut avoir de tangente perpendiculaire à l'axe des $x$, hormis aux points extrêmes dont les abscisses sont $+\frac{1}{0}$ et $-\frac{1}{0}$.

En effet, en différentiant l'équation générale ci-dessus, on trouve:

$$
\frac{d y}{d x}=m x^{m-1}+(m-1) A x^{m-2}+\cdots \cdots 2 K x+L
$$

pour l'expression de la tangente trigonométrique de l'angle que la touchante à la courbe fait avec l'axe des $x$, et puisque $m$ est un nombre entier positif, il s'ensuit que l'expression de $\frac{d y}{d x}$ ne peut devenir infinie pour aucun autre point, que pour ceux pour lesquels on a $x= \pm \frac{1}{0}$.

$4^{\circ}$. La courbe ne peut avoir aucun point de rebroussement, car d'abord, si la courbe avait un point de rebroussement dont la tangente fut inclinée ou parallèle à l'axe des $x$, il y aurait nécessairement des ordonnées qui couperaient la courbe en deux points, ce qui ne se peut vu la forme de l'équation; secondement il ne peut $\mathrm{y}$ avoir de point de rebroussement dont la tangente soit perpendiculaire à l'axe des $x$, puisque nous venons de prouver que la courbe ne peut avoir de tangentes de cetle direction.

$5^{\circ}$. Enfin, la courbe ne peut avoir de points multiples, puisque en de tels points, la courbe a deux tangentes, et il faudrait que $\frac{d y}{d x}$ puisse avoir deux valeurs différentes, pour une seule et même valeur de $x$, ce qui est évidemment impossible.

Il résulte de cela que la courbe ne saurait avoir d'autres points singuliers, que des points d'inflexion dont la tangente est inclinée ou parallèle à l'axe des $x$, mais jamais perpendiculaire, et, des points de maximum ou de minimum.

Après avoir ainsi examiné d'une manière générale les courbes qui correspondent à une équation d'un degré quelconque, examinons la différence caractéristique de celles, qui représentent les équations des degrés impairs 
de l'échelle des nombres, avec celles qui correspondent aux équations des degrés pairs.

Soit d'abord: $m=2 n+1$, c'est à dire un nombre impair quelconque: il est évident qu'en faisant: $x=\frac{1}{6}$, on aura aussi : $y=-\frac{1}{0}$, ce qui prouve que la courbe a une branche descendente qui se prolonge à l'infini dans la région des $(-\boldsymbol{X},-\boldsymbol{Y})$ désignant par là, la portion du tableau comprise entre les axes $\operatorname{des} x$ et celui des $y$ négatifs. - Mais si l'on suppose au contraire que $x$ devienne égal $\grave{a}+\frac{1}{0}$, on voit que $y$ devient aussi égal $\dot{a}+\frac{1}{\sigma}$, ce qui prouve que l'autre branche de la courbe est ascendante et s'étend à l'infini aussi, dans la région $(+\boldsymbol{X},+\boldsymbol{Y})$.

La courbe, correspondant à une équation du degré $2 n+1$, doit couper l'axe des $x$ en $2 n+1$ points, si l'on suppose que toutes les racines de l'équation sont réelles et inégales; - parconséquent il faut que cette courbe ait $n$ points de maximum, et $n$,points de minimum, comme on le voit dans la fig. 2 , qui représente la forme générale d'une courbe correspondante à une équation du $7^{\text {me }}$ degré pour laquelle on aurait $n=3$, et dont toutes les racines seraient réelles et inégales.

Celte courbe a comme on voit trois points de maximum: $m, m^{\prime \prime}, m^{1 v}$, et trois points de minimum: $m^{\prime}, m^{\prime \prime \prime}, m^{v}$, entre la branche descendante $p D$ et la branche ascendante $p^{\mathrm{vi}} \boldsymbol{A}$, et il est évident qu'il ne saurait $\mathrm{y}$ en avoir davantage, car alors l'axe des $x$ pourrait couper la courbe en plus de 7 points, ce qui est impossible, puisque l'équation n'a que 7 racines.

Il doit être bien entendu, que puisque l'on peut donner une infinité de valeurs différents aux coefficients d'une même équation, les sinuosités $p m p^{\prime}$, $\boldsymbol{p}^{\prime} \boldsymbol{m}^{\prime} \boldsymbol{p}^{\prime \prime}$, etc. peuvent aussi varier d'une infinité de manières différentes, et devenir, soit séparément, soit simultanément, extrêmement grandes ou extrêmement petites, égales ou inégales, et même nulles; - il peut arriver aussi que quelques unes des sinuosités au lieu de couper l'axe des $x$, ne fassent que le toucher, ou même s'en approchent sans le couper ni le toucher, et nous examinerons plus tard ces cas divers; - mais, aucune de ces sinuosités ne peut devenir infinie, puisque les coefficients de l'équation sont toujours des nombres finis.

J'avertis d'ailleurs, que dans ce qui précède comme dans ce qui va suivre, nous supposerons que les racines de l'équation sont toutes réelles et inégales, et que nous verrons plus tard de quelle manière la courbe caractérise les autres cas. 
Suppposons maintenant que l'equation soit d'un degré pair, $m=2 n$; en faisant: soit $x=-\frac{1}{0}$, soit $x=+\frac{1}{0}$, on trouve $y=+\frac{1}{0} ;-$ d'où il suit que la courbe a deux branches ascendentes, l'une dans la région $(+\boldsymbol{X},+\boldsymbol{Y})$ comme la courbe qui représente l'équation d'un degré impair, l'autre dans la région des $(-\boldsymbol{X},+\boldsymbol{Y})$ et qui remplace la branche descendente de la courbe du degré impair.

Cette courbe devant couper l'axe des $x$ en un nombre de points qui ne peut dépasser $2 n$, doit avoir entre ses deux branches ascendentes un nombre $n$ de points de minimum, et $n-1$ points de maximum, comme on peut le voir à la fig. 3 qui représente une équation du $8^{\text {ine }}$ degré, pour laquelle $n=4$; - tout ce que nous avons dit ci-dessus au sujet des variations que peuvent éprouver les sinuosités de la courbe d'un degré impair, convient également aux courbes des équations des degrés pairs.

Ainsi, les courbes des équations des degrés pairs, auront constamment deux branches ascendantes, qui s'étendront vers $+\frac{1}{0}$ et vers $-\frac{1}{0}$, et les courbes de degrés impairs auront constamment une seule branche ascendante qui s'étendra du côté des $x$ positifs jusqu'à $+\frac{1}{0}$, et une branche descendante qui s'étendra aussi jusqu'à $-\frac{1}{0}$ du côté des $x$ négatifs.

\section{VI.}

Après avoir examiné les formes générales et le genre de courbure qu'affectent les courbes correspondantes aux équations de tous les degrés, tant pairs qu'impairs, il est nécessaire de faire connaître d'autres propriétés générales des équations et des courbes correspondantes, sans lesquelles l'utilité de la formule (7.) deviendrait illusoire.

En effet, nous avons vu que la formule (7.) donne un résultat convergent, pourvu qu'entre l'extrêmité de l'abscisse arbitraire $(\alpha)$ qui sert de base à la série, et le point correspondant à la racine cherchée, il n'y ait aucun point singulier: - or, puisque la racine cherchée est inconnue, on ne peut juger à priori si la quantité $(\alpha)$ satisfait ou non à cette condition, et, comme l'équation a un nombre $m$ de racines, et qu'il faudra pour les déterminer faire sur la valeur de $(\alpha)$ un pareil nombre d'hypothèses différentes, l'incertitude de la condition se répettera chaque fois. - Il peut arriver encore, que deux, et même plusieurs des bypothèses que l'on pourrait faire sur $\alpha$, conduisent à la même racine de l'équation, et enfin, si par hazard la valeur 
attribuée à $\alpha$ était celle de l'abscisse d'un point de maximum ou de minimum, la série deviendrait infinie et ne donnerait aucune racine.

On voit donc qu'il est indispensable d'avoir une règle pour guider dans le choix des valeurs qu'il faut attribuer à la quantité arbitraire $\alpha$, afin d'être sur que chacune de ces valeurs donnera nécessairement l'une des différentes racines de l'équation; - en d'autres termes, il faut découvrir une règle par laquelle on puisse assigner les limites entre lesquelles il faut choisir les valeurs de $(\alpha)$, pour que chacune de ces valeurs donne la racine correspondante, au moyen de la formule (7.).

Pour cela examinons d'une manière générale les relations qui existent entre l'équation donnée:

$$
\boldsymbol{X}=\boldsymbol{x}^{m}+\boldsymbol{A} \boldsymbol{x}^{\mathrm{n}-1}+\cdots \boldsymbol{K} \boldsymbol{x}^{2}+\boldsymbol{L} \boldsymbol{x}+\boldsymbol{M}=\boldsymbol{y}
$$

et toutes les dérivées de cette équation, ou plûtôt entre toutes les courbes que ces équations représentent.

Ces équations dérivées sont:

$$
\begin{aligned}
& \boldsymbol{X}^{\prime}=m \boldsymbol{x}^{m-1}+(\boldsymbol{m}-1) \boldsymbol{A} \boldsymbol{x}^{m-2}+\cdots \cdots 2 \boldsymbol{K} \boldsymbol{x}+\boldsymbol{L}=y^{\prime}, \\
& \boldsymbol{X}^{\prime \prime}=\boldsymbol{m}(\boldsymbol{m}-1) \boldsymbol{x}^{m-2}+(\boldsymbol{m}-1)(\boldsymbol{m}-2) \boldsymbol{A} \boldsymbol{x}^{m-3}+\cdots \cdots 2 \boldsymbol{K}=y^{\prime \prime}, \\
& \quad \text { etc. etc. jusqu'à la dernière qui est } \\
& \boldsymbol{X}^{m}=\boldsymbol{m}(\boldsymbol{m}-1)(m-2)(m-3) \ldots \ldots(m-(m-2))(m-(m-1))=y^{m} \\
& =\text { Constante. }
\end{aligned}
$$

(La quantité $m$ dont $X^{m}$ et $y^{\iota^{n}}$ sont affectées, n'est que l'indice du rang de la dérivée et non une puissance.) Il est bien évident que la dernière dérivée $\boldsymbol{X}^{m}$, étant indépendante de la variable $x$, sera toujours positive, quel que soit la valeur de $x$, depuis $x=-\frac{1}{6}$ jusqu'à $x=+\frac{1}{0}$. - Mais, il n'en est pas de même des autres dérivées

$$
\boldsymbol{X}^{(m-1)}, \ldots \ldots \boldsymbol{X}^{\mathrm{Iv}}, \boldsymbol{X}^{\prime \prime \prime}, \boldsymbol{X}^{\prime \prime}, \boldsymbol{X}^{\prime \prime} \text { et de l'équation primitive } \boldsymbol{X},
$$

qui peuvent être tantôt positives, tantôt négatives, selon la valeur attribuée à $x$.

Si l'on fait dans les fonctions ci-dessus $x=-\frac{1}{b}$, il est évident que toutes celles où la puissance la plus élevée de $x$ sera un nombre pair, prendront le signe + , tandis que toutes les autres prendront le signe - . Si l'on fait au contraire $x=+\frac{1}{0}$, toutes les fonctions auront le signe + , quel que soit le degré de la fonction. - Ainsi en écrivant toutes ces fonctions en commençant par la dernière qui est indépendante de $x$, et dont le signe est toujours positif, nous aurons, en marquant au dessous de chaque fonction le 
15. F. Theremin, sur la résolution des équations.

signe qu'elle prendra, les deux suites:

(1.)

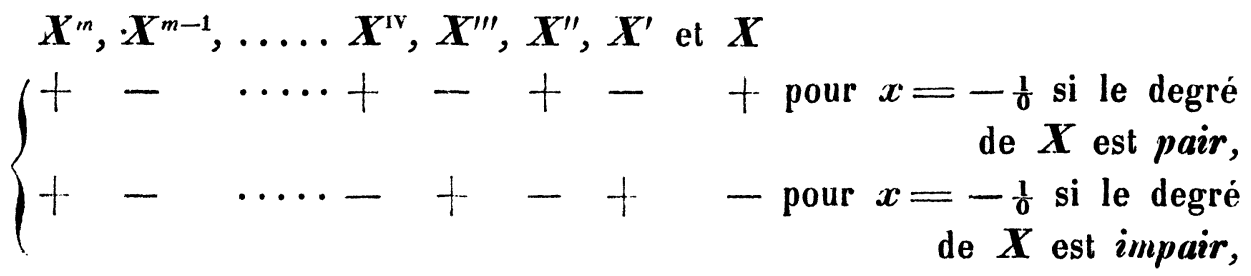

et

(2.)
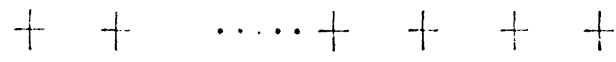
+ pour $x=+\frac{1}{d}$ quel que soit le degré de $x$.

Nous appellerons variation, ou changement de signe, la succession dans cette suite de deux signes différents, et permanence, la succession de deux signes semblables: - on voit que la suite supérieure n'a que des variations, tandis que la suite inférieure n'a que des permanences de signe, il suit donc de là, que dans le passage de la variable $x$, de la valeur $-\frac{1}{0}$ à la valeur $+\frac{1}{0}$, la suite des fonctions $\boldsymbol{X}, \boldsymbol{X}^{\prime}$, etc. perd autant de variations de signes, que l'équation du degré $m$ a de racines.

Nous allons prouver de plus, que la suite des fonctions ci-dessus ne perd pas toutes ses variations de signes à la fois, mais qu'elle n'en perd qu'une seule, chaque fois, que la variable $x$, en croissant continuellement depuis $-\frac{1}{0}$ jusqu'à $+\frac{1}{0}$, vient à atteindre et à dépasser une des racines de la proposée.

Pour cela, supposons que l'on construise les courbes correspondantes à l'équation donnée et a toutes ses dérivées, en représentant leurs ordonnées par $y, y^{\prime}, y^{\prime \prime}$, etc. nous aurons :

$$
\boldsymbol{X}=y, \quad \boldsymbol{X}^{\prime}=y^{\prime}, \quad \boldsymbol{X}^{\prime \prime}=y^{\prime \prime}, \quad \ldots \quad \boldsymbol{X}^{m}=y^{m} .
$$

Supposons aussi, qu'au lieu dé construire ces courbes sur le même axe, on les rapporte comme dans la (fig. 4), à des axes parallèles entre eux, $(-\boldsymbol{X},+\boldsymbol{X})$ pour la courbe correspondante à l'équation primitive' $\boldsymbol{X}=y ;\left(-\boldsymbol{X}^{\prime},+\boldsymbol{X}^{\prime}\right)$ pour la courbe correspondante à la première dérivée $X^{\prime}=y^{\prime}$, et ainsi de suite, en conservant d'ailleurs le même axe des ordonnées $(+\boldsymbol{Y},-\boldsymbol{Y})$ pour toutes ces courbes; - cela posé, examinons les variations de signes que subiront les ordonnées $y, y^{\prime}, y^{\prime \prime}$, etc. de ces diverses courbes, lorsqu'on fera varier l'abscisse commune $x$.

En examinant les courbes de la (fig. 4), on voit que les points d'intersection de l'axe avec l'une des courbes intermédiaires, correspondent aux 
points de maximum ou de minimum de la courbe précédente ou immédiatement supérieure, et que les points de maximum et de minimum de cette courbe correspondent aux points d'inflexion de la précédente, et aux points d'intersection de la suivante avec l'axe, et ainsi de suite. - De là d'abord une première conséquence: c'est que la première racine $O_{p}$ de la première courbe, du côté des $x$ négatifs, dépasse nécessairement de ce même côté la première racine de la seconde courbe, et parconséquent les premières racines de toutes les courbes suivantes ou dérivées; - et de même, la dernière racine $\boldsymbol{O}_{p}{ }^{\mathrm{IV}}$ de la première courbe, du côté des $x$ positifs, dépasse du même côté toutes les dernières racines des courbes dérivées. - Il est donc évident que si par un point $\alpha$, dont l'abscisse $\boldsymbol{O}_{\alpha}$ dépasse du côté négatif la première racine $\boldsymbol{O}_{\boldsymbol{p}}$ de la première courbe, on mène une perpendiculaire à l'axe des $x$, cette perpendiculaire prolongée indéfiniment ne coupera que la branche gauche de chaque courbe, à commencer par la première, quel que soit le degré de l'équation, et comme les courbes sont alternativement de degrés pairs et impairs, il s'ensuit que les ordonnées des courbes successives, pour le point $\alpha$, seront alternativement: négatives et positives, si comme dans la (figure 4), la première courbe est de degré impair, et au contraire positives et négatives, si la première courbe est de degré pair. - De même si l'on prend pour abscisse du côté des $x$ positifs une quantité $\boldsymbol{O} \beta$, qui dépasse la dernière racine de la première équation, on voit que les ordonnées de toutes les courbes correspondantes au point $\beta$, seront toutes positives, puisque la perpendiculaire indéfinie menée par ce point ne peut couper que les branches droites de toutes les courbes, qui sont toutes ascendantes.

Comme les points $\alpha$ et $\beta$ peuvent ètre aussi près qu'on voudra des points $p$ et $p^{\text {Iv }}$ qui déterminent les deux racines extrêmes de l'équation, il s'ensuit que ces deux racines $O p$ et $\boldsymbol{O}^{\mathrm{Iv}}$, sont deux limites telles: que toute valeur de $x$ prise en deça de la première, donnera pour $\boldsymbol{X}$ et ses dérivées autant de variations de signes, que l'équation donnée $\boldsymbol{X}=0$ a de racines, et, que toute valeur de $x$ qui surpassera la seconde limite, ne donnera pour $\boldsymbol{X}$ et ses dérivées que des permanences de signe; - parconséquent, on voit que la plus grande et la plus petite racine d'une équation, ou ce qui revient au même: que la plus grande racine positive et la plus grande racine négative, sont deux limites, entre lesquelles les valeurs de la variable $x$ doivent être comprises pour faire varier la suite des signes de la fonction $\boldsymbol{X}$ et de toutes ses dérivées; - en d'autres termes, toute valeur de $x$ qui ne serait pas 
comprise entre ces limites étant mise dans la fonction $\boldsymbol{X}$ et ses dérivées, ne peut donner que deux suites de signes, savoir: l'une qui n'aura que des variations de signe, si $x$ est moindre que la plus petite limite, et l'autre, que des permanences de signe, si $x$ surpasse la plus grande limite.

Nous avons vu que la première racine $\boldsymbol{O}_{p}$ de l'équation $\boldsymbol{X}=\mathbf{0}$, ou ce qui est la même chose, que l'abscisse $O p$ du premier point d'intersection de la première courbe $\boldsymbol{X}=y$ avec l'axe des $x$, dépassait du côté gauche toutes les premières racines des équations dérivées $X^{\prime}=0, X^{\prime \prime}=0$, etc. ou tous les premiers points d'intersection des courbes dérivées avec leurs axes respectifs, et que pour tout point $\alpha$ situé en deça du points $p$ on a la suite de signes:

$$
\begin{aligned}
& \boldsymbol{X}^{m}, \boldsymbol{X}^{m-1}, \ldots \ldots \boldsymbol{X}^{\mathrm{v}}, \boldsymbol{X}^{\prime \prime \prime}, \boldsymbol{X}^{\prime \prime}, \boldsymbol{X}^{\prime}, \boldsymbol{X} \\
& +\ldots \ldots-+\ldots \text { - }-\ldots \text { pour le point } \alpha
\end{aligned}
$$

dont le dernier à droite, c'est à dire celui qui correspond à la première courbe est négatif, puisque la première courbe dans la figure (4) représente une équation de degré impair. - Mais on voit que si le point $\alpha$ en s'avançant de gauche à droite venait à atteindre le point $p$, on aurait en ce point $\boldsymbol{X}=\boldsymbol{y}=0$, puisque l'abscisse $\boldsymbol{O} \boldsymbol{p}$ est la première racine de l'équation donnée, et que si le point continuait à s'avancer jusqu'en $\alpha^{\prime}$ dont l'abscisse est moindre que $O_{p}$, la fonction $\boldsymbol{X}$ changerait de signe, puisque l'ordonnée de la première courbe au point $\alpha^{\prime}$ est positive; - ainsi la suite des signes ci-dessus se changerait en :

$$
\begin{aligned}
& \boldsymbol{X}^{m}, \boldsymbol{X}^{m-1}, \ldots \ldots \boldsymbol{X}^{1 v}, \boldsymbol{X}^{\prime \prime \prime}, \boldsymbol{X}^{\prime \prime}, \boldsymbol{X}^{\prime}, \boldsymbol{X} \\
& +-\ldots \ldots-+-1+\text { pour le point } \alpha^{\prime} .
\end{aligned}
$$

Et comme le point $\alpha^{\prime}$ peut être aussi près qu'on voudra du point $p$, il s'en suit que la suite des signes, qui avait autant de variations que l'équation donnée a de racines, a perdu une variation et gagné une permanence de signe, aussitôt que la variable a dépassé la première racine.

Il est bien évident que, tant que le point $\alpha^{\prime}$ en continuant de s'avancer vers la droite n'aura pas dépassé la première racine $q$ de la seconde courbe, il n'y aura aucun changement dans la suite des signes que nous venons de trouver pour le point $\alpha^{\prime}$; mais pour savoir ce que devient cette suite quand le point $\alpha^{\prime}$ aura dépassé la racine $q$ et sera en $\alpha^{\prime \prime}$, considérons séparément les deux suites de signes pour ces deux points, et relativement à l'équation $\boldsymbol{X}^{\prime}=\mathbf{0}$ ou à la première dérivée seulement, nous aurons: 


$$
\begin{aligned}
& \boldsymbol{X}^{m}, \boldsymbol{X}^{m-1}, \ldots \ldots \boldsymbol{X}^{\mathrm{v}}, \boldsymbol{X}^{\prime \prime \prime}, \boldsymbol{X}^{\prime \prime}, \boldsymbol{X}^{\prime} \\
& +-\cdots \cdots-+-+ \text { pour le point } \alpha^{\prime} \text {, } \\
& +-\cdots \cdot-+- \text { pour le point } \alpha^{\prime \prime} \text {, }
\end{aligned}
$$

puisque la variable $x$, en passant de $\alpha^{\prime} \dot{a} \alpha^{\prime \prime}$, a dépassé la première racine de $X^{\prime}=0$; - or en ajoutant à ces deux suites, du côté droit, le signe de l'ordonnée de la première courbe pour chacun de ces points, qui est t, on aura :

$$
\begin{aligned}
& \boldsymbol{X}^{m}, \boldsymbol{X}^{m-1}, \ldots \ldots \boldsymbol{X}^{\mathrm{iv}}, \boldsymbol{X}^{\prime \prime \prime}, \boldsymbol{X}^{\prime \prime}, \boldsymbol{X}^{\prime}, \boldsymbol{X} \\
& +-\cdots \cdots-+-++ \text { pour le point } \alpha^{\prime} \text {, } \\
& +-\cdots \cdot-+-\quad+\text { pour le point } \alpha^{\prime \prime} \text {. }
\end{aligned}
$$

Ce qui fait voir que même après avoir dépassé la première racine de la seconde courbe, le nombre des variations et celui des permanences de signe n'a pas changé, seulement la permanence a changé de place dans la suite des signes, puisque la permanence de la suite supérieure ++ , s'est changée en une variation -+ , et que' la variation -+ s'est changée en une permanence - -

Enfin, en considérant la première racine de la troisième courbe, ou de la seconde dérivée $X^{\prime \prime}=0$, on voit qu'en faisant passer la variable $x$ d'un côté à l'autre du point $r$, ou de $\alpha^{\prime \prime}$ en $\alpha^{\prime \prime \prime}$, on aura en considérant seulement les suites des signes à partir de la troisième courbe,

$$
\begin{aligned}
& \boldsymbol{X}^{m}, \boldsymbol{X}^{m-1}, \ldots \ldots \boldsymbol{X}^{\mathrm{iv}}, \boldsymbol{X}^{\prime \prime \prime}, \boldsymbol{X}^{\prime \prime} \\
& +\quad-\ldots \ldots-+- \text { pour le point } \alpha^{\prime \prime}, \\
& +-\ldots \ldots-++ \text { pour le point } \alpha^{\prime \prime \prime} .
\end{aligned}
$$

Mais en ajoutant à droite de ces deux suites les signes des ordonnées $y^{\prime}$ et $y$, pour ces deux points, qui sont tout deux - pour $y^{\prime}$ et tout deux + pour $y$, on aura:

$$
\begin{aligned}
& \boldsymbol{X}^{m}, \boldsymbol{X}^{m-1}, \ldots \ldots \boldsymbol{X}^{\mathrm{vv}}, \boldsymbol{X}^{\prime \prime \prime}, \boldsymbol{X}^{\prime \prime}, \boldsymbol{X}^{\prime}, \boldsymbol{X} \\
& +\ldots \ldots-+ \text { - }-\ldots u r \alpha^{\prime \prime}, \\
& +-\ldots \ldots-+ \text { + }+ \text { - pour } \alpha^{\prime \prime \prime}
\end{aligned}
$$

d'où l'on voit, que même en dépassant la première racine de la troisième courbe, le nombre des variations et des permanences dans la suite des signes n'a pas changé, seulement la permanence - - qui était au second rang, à passé au troisième où l'on trouve une permanence $t+d$ dans la suite qui correspond au point $\alpha^{\prime \prime \prime}$. 
Or, le point $\alpha^{\prime \prime \prime}$ peut être pris aussi près qu'on voudra du point $\boldsymbol{p}^{\prime}$, qui indique la seconde racine de l'équation de la première courbe, ainsi, en résumant cette analyse on voit que:

$1^{\circ}$. La suite des signes des fonctions $X, X^{\prime}$, etc. perd une variation et gagne une permanence de signe, dès que la valeur attribuée à $x$ a dépassé la première racine de l'équation, et que:

$2^{\circ}$. Le nombre total des variations, et le nombre total des permanences restent les mêmes, tant que $x$ ne sort pas de l'intervalle $p p^{\prime}$ de la première racine à la seconde.

Cela prouvé, il est évident qu'aussitôt que la variable $x$ aura dépassé la seconde racine $\boldsymbol{O}_{p^{\prime}}$ de la première équation, ou que le point $\alpha^{\prime \prime \prime}$ se sera avancé jusqu'en $\alpha^{\mathrm{Iv}}$, l'ordonnée $y$, deviendra de nouveau négative, et les suites des signes correspondantes à ces deux points seront:

$$
\begin{aligned}
& \boldsymbol{X}^{m}, \boldsymbol{X}^{m-1}, \ldots \ldots \boldsymbol{X}^{\mathrm{iv}}, \boldsymbol{X}^{\prime \prime \prime}, \boldsymbol{X}^{\prime \prime}, \boldsymbol{X}^{\prime}, \boldsymbol{X} \\
& +-\cdots \cdots-+\quad+\quad+\text { pour le point } \alpha^{\prime \prime \prime} \text {, } \\
& +-\cdots \cdots-++\cdots-\text { pour le point } \alpha^{\mathrm{IV}} \text {. }
\end{aligned}
$$

Ce qui fait voir, qu'en dépassant de gauche à droite la second racine de l'équation donnée, la variable $x$ fait perdre une seconde variation de signes à la suite des fonctions $\boldsymbol{X}, \boldsymbol{X}^{\prime}, \boldsymbol{X}^{\prime \prime}$, etc. et fait gagner à celte suite une seconde permanence.

En continuant cette analyse des signes que doivent prendre les fonctions, ou les ordonnées des courbes, entre la seconde et la troisième racine, puis entre la troisième et lá quatrième, et ainsi de suite, on s'assurerait aisément que dans l'intervalle de deux racines de la première équation, la suite des signes conserve invariablement le même nombre de variations et le même nombre de permanences de signes, et qu'au contraire la suite perd une variation et gagne une permanence chaque fois que la variable passe d'un intervalle à un autre de gauche à droite, c'est à dire qu'elle dépasse dans ce sens l'une des racines de la proposée.

Il est aisé de voir, que si la première courbe eut été d'un degré pair, l'analyse des signes eut été entièrement semblable à celle que nous venons de faire, seulement le signe du polynôme $\boldsymbol{X}$ qui était négatif dans la première suite, eut été positif; - on voit aussi qu'au lieu de commencer cette analyse des signes en faisant varier $x$ de gauche à droite, on aurait pu faire varier $x$ de droite à gauche en commençant par le point $b$, (fig. 4) qui dépasse 
toutes les racines du côté droit, et pour lequel la suite des signes n'a aucune variation, mais qui en gagne une aucontraire chaque fois que $x$, en décroissant vient à dépasser une des racines de droite à gauche, comme on peut le voir pour le point $\beta^{\prime}$ situé entre la dernière et l'avant dernière des racines de l'équation proposée.'

Cette propriété des racines, de faire perdre une variation de signes a la suite des fonctions $\boldsymbol{X}, \boldsymbol{X}^{\prime}, \boldsymbol{X}^{\prime \prime}$, etc. chaque fois que $x$ vient à dépasser une de ces racines, a été prise par Fourrier pour bâse de la méthode qu'il a crée pour la recherche des racines des équations de tous les degrés. Quoique je me propose d'établir une méthode différente, je n'en ai pas moins crû devoir exposer la démonstration du théorème de Fourrier, tant pour la démonstration, que je crois nouvelle, que pour le théorème en lui-même, qui est un remarquable phénomène mathématique.

\section{VII.}

Fig. 5. Soil: $m \boldsymbol{I}^{v} \boldsymbol{I}^{\prime} \boldsymbol{I I}^{\prime \prime} \boldsymbol{I}^{\prime \prime \prime} \boldsymbol{I}^{\mathrm{v}} \boldsymbol{m}^{\prime}$, une portion de la courbe qui représente une équation d'un degré quelconque, portion qui est comprisè entre un point de minimum $m$ et un point de maximum $m$, et supposons qu'il s'agisse de déterminer la racine $\boldsymbol{O}_{\boldsymbol{p}}$ qui correspond à cette partie de la courbe.

On voit du premier coup d'oeil, que: soit que l'axe des $x$ passe au dessus du point d'inflexion $I$, soit qu'il passe au dessous comme dans la (fig. 5), le point satisfait à la condition nécessaire à la convergence de la formule (7.), si on le prend pour point de départ de l'approximation, puisqu'entre ce point, et le point $p$ qui détermine la racine cherchée, la courbe ne présente aucun point singulier; - de plus, tous les points sitnés comme $\boldsymbol{I}^{\prime}$ entre la racine $\boldsymbol{p}$ et le point d'inflexion $I$, étant encore plus proches de la racine, seront encore plus favorables à l'approximation de cette dernière, que le point I lui même. Quant aux points situés au delà du point d'inflexion, par rapport à la racine, tels que le sont les points $I^{\prime \prime}$ et $\boldsymbol{I}^{\prime \prime \prime}$, si la touchante à la courbe en ces points, tombe entre la racine et la touchante au point d'inflexion, comme cela arrive pour le point $I^{\prime \prime}$, ou même si cette touchante ne dépasse la racine que d'une faible quantité, comme celle du point $I^{\prime \prime \prime}$, et de manière à ramener l'approximation dans l'espace $p n$ : tous ces points peuvent être pris pour point de départ de l'approximation, et en prenant l'abscisse de l'un de ces points pour la quantité arbitraire $\alpha$ de la formule (7.) on arrivera infailliblement à la racine cherchée $O_{p}$. 
Pour d'autres points tels que $\boldsymbol{I}^{\mathrm{iv}}$ ou $\boldsymbol{I}^{\mathrm{v}}$, situés plus près des points $m$ et $m^{\prime}$, la touchante à la courbe en ces points, ferait sortir l'approximation de la portion de courbe examinée, et il arriverait que le résultat au lieu de se rapprocher de la racine cherchée $O_{p}$, s'en éloignerait, et s'approcherait d'une autre racine, située sur une autre portion de la courbe.

On voit donc, que non seulement le point d'inflexion $\boldsymbol{I}$, mais encore tous les points pris sur une certaine étendue de la courbe de part et d'autre de ce point, peuvent servir de point de départ pour conduire à la racine cherchée $O p$, et que parconséquent toute valeur seulement approchée de l'abscisse On du point d'inflexion, conduira nécessairement à la racine correspondante.

Il suit de là une première conséquence: c'est, qu'une courbe correspondante à une équation du degré $m$, ayant $(m-1)$ points de maximum et de minimum, et parconséquent $(m-2)$ points d'inflexion, il faut commencer par déterminer les abscisses de ces $m-2$ points d'inflexion, et prendre les valeurs approchées de chacune d'elles pour bâse de l'approximation des (m-2) racines correspondantes de l'équation donnée. - Quand on sera parvenu par ce moyen à déterminer $(m-2)$ racines de la proposée, on trouvera facilement les deux racines restantes ou extrêmes de l'équation donnée, au moyen d'une équation du $2^{\text {d }}$ degré dont on formera les coefficients, d'une part en retranchant du coefficient du $2^{d}$ terme de la proposée la somme des $m-2$ racines déjà trouvées, et d'autre part en divisant le dernier terme par le produit de ces mêmes racines. (Il est clair que la formation d'une équation du $2^{\mathrm{d}}$ degré pour déterminer les deux dernières racines, n'est praticable que dans le cas où toutes les racines de la proposée sont réelles, ce dont j'ai d'ailleurs expressément averti. - Nous verrons plus tard comment le procédé se modifie quand les racines deviennent imaginaires ou manquantes.)

De cette manière la question se trouve ramenée à trouver les abscisses des (m-2) points d'inflexion de la courbe qui représente l'équation donnée; mais, nous avons vu, que les points d'inflexion de la courbe primitive, correspondent aux points de maximum et de minimum de la courbe qui représente la première dérivée, et parconséquent aux racines de la $2^{\text {de }}$ dérivée; - ainsi, la recherche des racines de l'équation du degré $m$, se trouve ramenée à la recherche des racines de la seconde dérivée qui n'est plus que du degré $(x-2)$, et qui se trouvera, par des considérations semblables ramenée à la recherche des racines d'une équation d'un degré $(m-4)$, puis à celles d'une équation 
d'un degré $m-6$, et jusqu'à ce qu'enfin l'on arrive à une équalion du $2^{\text {d }}$ ou du $1^{\text {er }}$ degré, selon que la plus haute puissance de $x$ dans la proposée est pair ou impair.

Pour donner un exemple de la méthode proposée, cherchons les racines de l'équation:

$$
X=x^{3}-5 x^{2}-29 x+105=0
$$

qui sont toutes trois réelles, et égales aux nombres: $-5,+3,+7$.

Les dérivées de cette équation seront:

$$
\begin{aligned}
& X^{\prime}=+3 x^{2}-10 x-29 \\
& X^{\prime \prime}=+6 x-10, \\
& X^{\prime \prime \prime}=+6 .
\end{aligned}
$$

Traçons sur un tableau séparé, fig. 6, trois lignes horizontales et parallèles entr'elles, $(-\boldsymbol{X},+\boldsymbol{X}),\left(-\boldsymbol{X}^{\prime},+\boldsymbol{X}^{\prime}\right)$ et $\left(-\boldsymbol{X}^{\prime \prime},+\boldsymbol{X}^{\prime \prime}\right)$ que nous prendrons pour axes des $x$ respectifs des trois lieux géométriques, de l'équation donnée et de ses deux premières dérivées $\boldsymbol{X}^{\prime}$ et $\boldsymbol{X}^{\prime \prime}$, et traçons aussi une ligne $(+\boldsymbol{Y},-\boldsymbol{Y})$ perpendiculaire $\dot{a}$ ces trois axes, et que nous prendrons pour axe commun des $y$.

En posant d'abord:

$$
X^{\prime \prime}=+6 x-10=0 .
$$

Nous trouvons

$$
\text { (a.) } \quad x=+1,666 \ldots
$$

pour la valeur de la racine de la seconde dérivée, qui sera en même temps l'abscisse du point minimum de la courbe correspondante à la première dérivée et parconséquent celle du point d'inflexion de la courbe qui correspond à l'équation donnée. Portons donc sur l'axe $\left(-\boldsymbol{X}^{\prime \prime},+\boldsymbol{X}^{\prime \prime}\right)$ à partir de l'origine $\boldsymbol{O}^{\prime \prime}$ et du côté positif une ligne $O^{\prime \prime} n^{\prime \prime}=+1,666 \ldots$ nous aurons cette abscisse commune; - de plus par le point $n^{\prime \prime}$ élevons la perpendiculaire indéfinie $n^{\prime \prime} k$, nous aurons: $O n=O^{\prime} n^{\prime}=O^{\prime \prime} n^{\prime \prime}$.

Substituons la valeur trouvée pour $x$ dans la première dérivée $\boldsymbol{X}^{\prime}$ el dans l'équation donnée $\boldsymbol{X}$, nous trouvons:

$$
\begin{aligned}
& \boldsymbol{X}_{(+1,666 \ldots)}^{\prime}=-42,2444, \ldots \\
& \boldsymbol{X}_{(+1,666 \ldots)}=+48,990276 \ldots
\end{aligned}
$$

La première de ces valeurs prouve que l'ordonnée du point minimum de la première dérivée est égale ’̀ $-42,2444 \ldots ;-$ construisons ce point en 
portant sur la perpendiculaire $n^{\prime \prime} k$, a partir de l'axe $\left(-\boldsymbol{X}^{\prime},+\boldsymbol{X}^{\prime}\right)$ et du côté des $y$ négatifs, la ligne $n^{\prime} m=-42,244 \ldots$

La seconde des valeurs ci-dessus prouve que le point d'inflexion de la courbe qui correspond à l'équation donnée a pour ordonnée $+48,990276 \ldots$; construisons aussi ce point en prenant $n I=+48,990276 \ldots$

Présentement cherchons les racines de la première dérivée qui étant du second degré se resoud par le procédé ordinaire, en posant:

$$
X^{\prime}=3 x^{2}-10 x-29=0,
$$

nous trouvons pour ces racines: $-1,861 \ldots$ et $+5,861 \ldots$ construisons ces points en portant sur l'axe $\left(-\boldsymbol{X}^{\prime},+\boldsymbol{X}^{\prime}\right)$ les abscisses:

$$
O^{\prime} p=-1,861 \ldots \text { et } \quad O^{\prime} p^{\prime}=+5,861 \ldots
$$

et menons par les points $\boldsymbol{p}$ et $\boldsymbol{p}^{\prime}$ les perpendiculaires $\boldsymbol{p} \boldsymbol{k}^{\prime}$ et $\boldsymbol{p}^{\prime} \boldsymbol{k}^{\prime \prime}$, qui couperont l'axe des $(-\boldsymbol{X},+\boldsymbol{X})$ aux points $q$ et $q^{\prime} ;-\operatorname{ces}$ deux abscisses $\boldsymbol{O}^{\prime} \boldsymbol{p}$ et $\boldsymbol{O}^{\prime} \boldsymbol{p}^{\prime}$, sont aussi celles des points maximum et minimum de la courbe correspondante à l'équation donnée $\boldsymbol{X}=0$, et en mettant les valeurs de ces abscisses dans le polynome $\boldsymbol{X}$, on aura:

$$
\boldsymbol{X}_{(-1,861)}=+135,168 \ldots, \quad \boldsymbol{X}_{(5,861)}=-36,288 \ldots
$$

La première de ces deux valeurs, prouve que l'ordonnée du point de maximum, qui correspond à l'abscisse $O q$, est positive et égale à $+135,168 \ldots$ et nous la construisons en prenant $q \boldsymbol{m}^{\prime}$ égal à ce nombre; - la seconde valeur prouve que la courbe a un point de minimum, correspondanl à l'abscisse $O q^{\prime}$, dont l'ordonnée est négative, et que nous construirons en prenant $q^{\prime} m^{\prime \prime}=-36,288 \ldots$

Il suit de ceci, que la courbe qui est le lieu géométrique de l'équation donnée, coupe nécessairement l'axe des $x$ en trois points, et que parconséquent toutes les 3 racines de l'équation sont réelles et inégales. - En effet, la courbe élant de degré impair, aura du côté négatif une branche descendante, indéfinie, et qui partira du point de maximum $m^{\prime}$ situé au dessus de l'axe, que celte branche doit parconséquent couper; - de même, la courbe ayant du côté des $x$ positifs une branche ascendante indéfinie, et qui part du point de minimum $\cdot m^{\prime}$ situé au dessous de l'axe, il est évident que cette branche ascendante doit aussi nécessairement couper l'axe; - enfin la portion de courbe qui s'ètend du point $m^{\prime}$ au point $\boldsymbol{m}^{\prime \prime}$, doit aussi nécessairement couper l'axe puisque ces deux points sont situés des deux côlés opposés de cet axe.

Crelle's Journal f. d. M. Bd. XLIX. Heft 3.

29 
Après nous être assuré que les trois racinès de l'équation sont réelles, il ne nous reste plus qu'à appliquer la valeur $(\alpha$.) à la recherche de la racine qui correspond au point d'inflexion.

En mettant donc la valeur $(\alpha$.$) qui est +1,666 \ldots$ au lieu de $x$ dans les polynômes $\boldsymbol{X}$ et $\boldsymbol{X}^{\prime}$, nous aurons pour première approximation;

$x=+1,666 \ldots-\left\{\frac{X_{(+1,666)}}{X_{(+1,666 \ldots)}^{\prime}}\right\}=+1,666-\left\{\frac{+48,990276 \ldots}{-42,244444 \ldots}\right\}=+2,826334 \ldots$ En prenant cette première approximation, avec deux chiffres décimaux pour bâse d'une seconde approximation, nous aurons:

$$
x=+2,826334 \ldots-\left\{\frac{X_{(+2,82)}}{X_{(+2,82)}^{\prime}}\right\}=+2,29976556 \ldots
$$

En prenant cette nouvelle valeur avec trois chiffres décimaux pour bàse d'une troisième approximation, nous aurons:

$$
x=+2,9976556 \ldots-\left\{\frac{X_{(+2,997)}}{X_{(+2,997)}^{\prime}}\right\}=+2,999999 \ldots
$$

nombre qui ne diffère de +3 que de la millionième partie de l'unité.

D'après la situation du point d'inflexion et de la courbe, quelque loin que l'on pousse l'approximation, le résultat sera toujours moindre que la racine cherchée, d'où il suit, qu'en augmentant de l'unité le dernier chiffre trouvé de la partie décimale de la racine, il doit arriver de deux choses l'une: $1^{\circ}$ ou l'on tombera sur la vraie valeur de la racine, ou: $2^{\circ}$ l'on dépassera cette valeur. - Dans le premier cas, en substituant la valeur ainsi augmentée, au lieu de $x$, dans l'équation donnée, cette équation sera satisfaite et le résultat égale à zéro. - Dans le second cas, l'équation ne sera pas satisfaite, mais le résultat sera de signe contraire à celui que donne l'approximation trouvée, ce qui prouve que la racine est dépassée par l'augmentation d'une unité décimale qu'on a ajoutée au dernier chiffre.

Ajoutant donc une unité au dernier chiffe décimal de l'approximation trouvée, on obtient $x=+3$, valeur qui tombe dans le premier cas et qui satisfait à l'équation.

Pour trouver les deux racines restantes, retranchons le nombre trouvé +3 , du coefficient du second terme de l'équation donnée, pris avec un signe contraire, et qui est ici +5 , nous aurons $+5-3=+2$ pour la somme des deux racines restantes; - divisant ensuite par +3 le dernier terme de l'équation donnée, pris avec un signe contraire, qui sera ici -105, on trouve - 35 pour le produit des deux racines restante, qui seront parconséquent 
données par l'équation:

d'où l'on tire:

$$
x^{\prime 2}-2 x^{\prime}-35=0
$$

$$
x^{\prime}=+1 \pm 136
$$

qui donne. -5 et +7 pour les deux racines cherchées.

Il faut observer que chaque fois que la racine cherchée sera un nombre entier, ou au moins une quantité exprimable exactement par un nombre fini de chiffres décimaux, il arrivera: $1^{\circ}$. Que si l'approximation est au dessous de la racine cherchée, on obtiendra tous les chiffres exacts de la valeur de cette racine, moins le dernier, qui sera d'une unité moindre, mais suivi d'un nombre de 9, d'autant plus grand qu'on aura poussé l'approximation plus loin. $2^{\circ}$. Si au contraire l'approximation donne des valeurs plus grandes que la racine, on retrouvera tous les chiffres de cette racine, et ce nombre sera séparé du reste des chiffres significatifs de la décimale par un nombre d'autant plus grand de zéros qu'on se sera plus approchée de la racine. - Dans le premier cas on ajoute une unité au dernier neuf de la fraction décimale, et dans le second cas on rejette les derniers chiffres séparés par les zéros, et l'on examine si le résultat satisfait exactement à l'équation.

Il faut encore observer qu'en faisant cette analyse pour ainsi dire géométrique de l'équation et de ses dérivées, il n'y a nulle nécessité en déterminant les points singuliers de chaque courbe, que les abscisses et les ordonnées soyent indiquées avec une grande précision; - il suffit de les tracer à la main, en conservant aux points extrêmes des abscisses leur relation de position respective. - Il en est de même pour les ordonnées, qui sont en général extrêmement grandes par rapport aux abscisses; il suffit d'indiquer si ces ordonnées sont positives ou négatives, et dans le cas de deux ou plusieurs ordonnées de même signe, la figure doit indiquer laquelle de deux ordonnées consécutives est plus grande, ou plus petite, afin que la courbe qu'on ferait passer par leurs extrêmités, présente les mêmes phénomènes de courbure, que ceux qui caractériseraient la courbe, en la supposant construite d'après les proportions de l'échelle.

Soit en effet:

et

$$
\boldsymbol{x}^{m}+\boldsymbol{A} \boldsymbol{x}^{m-1}+\cdots \boldsymbol{L} \boldsymbol{x}+\boldsymbol{M}=\boldsymbol{y}
$$

$$
\boldsymbol{x}^{m}+\boldsymbol{A} \boldsymbol{x}^{m-1}+\cdots \boldsymbol{L} \boldsymbol{L}+\boldsymbol{M}=\boldsymbol{n} \boldsymbol{y}^{\prime}
$$

les équations de deux courbes; - il est de toute évidence que ces deux 
courbes coupent l'axe des $x$ aux mêmes points, et que les abscisses des points d'inflexion, de maximum et de minimum, sont les mêmes pour les deux courbes, et cependant les ordonnées correspondantes aux deux courbes, pour une même abscisse, sont dans le rapport de $n$ à 1 .

Cette méthode, de tracer les courhes correspondantes à l'équation et à ses dérivées en commençant par ces dernières, a l'avantage de faciliter les opérations en indiquant les phénomènes de courbure de chaque ligne, et surtout, si l'on a soin d'inscrire sur le tableau, les valeurs des abscisses, celles des ordonnées de points singuliers, et les racines des courbes, à mésure qu'on les détermine.

Les racines des équations dérivées ne servant que comme quantités auxiliaires, il est inutile dans le plus grand nombre de cas de pousser loin leur approximation, et l'on peul en général se contenter d'un ou deux chiffres décimaux exacts; quant aux racines de l'équation primitive, ou donnée, on poussera l'approximation aussi loin qu'on voudra, ou aussi loin que pourra l'exiger la nature du problême.

\section{VIII.}

Dans tout ce que nous avons vu jusqu'à présent, nous avons supposé, à dessein, que toutes les racines de l'équation proposée étaient réelles et inégales, et cette supposition constitue le cas le plus général. - Il nous reste donc pour compléter notre méthode, à examiner le cas particulier des racines égales, et celui des racines imaginaires ou manquantes.

Pour cela, analysons d'abord les variations qui peuvent être produites dans le lieu géométrique d'une équation, soit relativement à sa position par rapport aux axes des coordonnées, soit relativement aux phénomènes de courbure qui le caractérisent, sans changer le degré de l'équation.

Soit, pour fixer les idées, le lieu géométrique d'une équation du $5^{\mathrm{mr}}$ degré, dont toutes les racines sont réelles et inégales, comme on le voit dans la (fig. (7) et dont $(+\boldsymbol{X},-\boldsymbol{X})$ est l'axe des abscisses, et $(+\boldsymbol{Y},-\boldsymbol{Y})$ celui des ordonnées.

Il est évident qu'en quelque lieu que l'on transporte les axes des coordonnées, la courbe ne changera pas, mais les constantes de l'équation varieront, et parconséquent ses racines varieront aussi.

Supposons d'abord qu'on transporte l'origine des abscisses $\boldsymbol{O}$ en un point quelconque $\boldsymbol{P}$ pris sur l'axe des abscisses, comme on le voit dans la 
(fig. 7); - il est clair que les racines de l'équation qui étaient $O r, O s, O t$, $\boldsymbol{O}_{u}$ et $\boldsymbol{O}_{v}$, sont devenus $\boldsymbol{P r}, \boldsymbol{P} s, \boldsymbol{P}, \boldsymbol{P} u$ et $\boldsymbol{P} v$, et ne diffèrent des premières que de la quantité $\boldsymbol{O P}$ dont on a transporté l'axe des ordonnées, ou l'origine des abscisses. - On voit que ce genre de transformation fait varier à la fois toutes les constantes de l'équation, mais ne change en rien la nature des racines dont les différences respectives restent invariable, mais qui ne peuvent devenir par là ni égales ni imaginaires. - On voit aussi que par une transformation de ce genre on peut rendre toutes les racines de l'équation, soit positives, soit négatives, si l'on avait besoin d'une telle transformation.

Supposons ensuite que ce soit l'origine des ordonnées, ou l'axe des abscisses, que l'on transporte parallèlement à lui même, successivement de $O$ en $O^{\prime}, O^{\prime \prime}, O^{\prime \prime \prime}, O^{\prime v}$, etc. - On voit d'abord que .tant que le nouvel axe des abscisses $\left(-\boldsymbol{X}^{\prime},+\boldsymbol{X}^{\prime}\right)$ n'aura pas dépassé au moins l'un des points de maximum in et $\boldsymbol{n}^{\prime \prime}$, cet axe ne cessera pas de couper la courbe en cinq points, comme dans sa position primitive, et que l'équation ne cessera pas d'avoir cinq racines réelles et inégales, avec cette différence pourtant, que plus l'axe s'approche des points de maximum $m$ el $m^{\prime \prime}$, et plus les deux racines les plus voisines de ces points tendront à se rapprocher. - Quand le nouvel axe viendra à se confondre avec la tangente $\boldsymbol{T}^{\prime \prime} \boldsymbol{T}^{\prime \prime}$ du point maximum le moins élevé $\boldsymbol{u}^{\prime \prime}$, comme on le voit dans la figure en $\left(-\boldsymbol{X}^{\prime \prime},+\boldsymbol{X}^{\prime \prime}\right)$ les deux racines les plus voisines de ce point, et qui étaient d'abord $O \boldsymbol{t}$ et $\boldsymbol{O} \boldsymbol{u}$, puis qui étaient devenues $O^{\prime} t^{\prime}$ et $O^{\prime} u^{\prime}$, sont enfin devenues égales entr'elles et à $O^{\prime \prime} m^{\prime \prime}$, et les deux points d'intersection de la sinuosité $t m^{\prime \prime} u$ avec l'axe des $x$, se sont réunis au point de maximum $m^{\prime \prime}$, pour former un point de contact avec le nouvel axe des $x$.

Si l'axe des $x$ continuait à s'élever jusqu'aux points $\boldsymbol{O}^{\prime \prime \prime}, \boldsymbol{O}^{\text {iv }}$, comme on le voit dans la figure, il dépasserait d'abord le point $m^{\prime \prime}$, et il n'y aurait plus de point de conlact, parconséquent les deux racines qui s'élaient réunies au point $m^{\prime \prime}$ en devenant égales, viendraient à manquer, on seraient imaginaires.

Dans la position de l'axe indiquée dans la figure: en $\left(-\boldsymbol{X}^{\prime \prime \prime},+\boldsymbol{X}^{\prime \prime \prime}\right)$ le nouvel axe touche la courbe au point $m$, et l'équation de la courbe correspondante à ce nouvel axe, aurait deux racines égales $\boldsymbol{O}^{\prime \prime \prime} m$, deux racines imaginaires manquantes au dessus du point $m^{\prime \prime}$ dépassé par l'axe, et enfin une troisième racine réelle $\boldsymbol{O}^{\prime \prime \prime} \boldsymbol{v}^{\prime \prime}$. - - Dans la position de l'axe indiquée dans la figure en $\left(-\boldsymbol{X}^{\mathrm{w}},+\boldsymbol{X}^{\mathrm{iv}}\right)$, les points de maximum $m$ et $\boldsymbol{m}^{\prime \prime}$ sont tout deux 
dépassés par cet axe, et la courbe aura évidemment quatre racines imaginaires, et une seule racine réelle $\boldsymbol{O}^{\mathrm{iv}} \boldsymbol{v}^{\mathrm{iv}}$.

Il est évident, que si l'axe des $x$ aulieu de s'élever au dessus de sa position primitive, s'abaissait au dessous parallèlement à lui même, les mêmes phénomènes se produiraient, et enfin quand l'axe aurait dépassé les deux points de minimum $m^{\prime}$ et $\boldsymbol{m}^{\prime \prime \prime}$, comme on le voit en $\left(-\boldsymbol{X}^{\mathbf{v}},+\boldsymbol{X}^{\mathbf{v}}\right)$, il n'y aurait plus qu'une racine réelle $\boldsymbol{O}^{v} \boldsymbol{r}^{\mathrm{v}}$, et quatre racines imaginaires.

Il faut remarquer, que tous ces changements s'obtiennent en faisant seulement varier le dernier terme de l'équation, c'est à dire celui qui est indépendant de $x$, et que parconséquent cette variation n'affecle en rien les coefficients des puissances de l'inconnue.

Dans les deux cas que nous venons d'examiner, nous avons successivement changé la position des axes relativement au lieu géométrique de l'équation, supposé fixe, ce qui est la même chose que si nous avions transporté la courbe, en supposant fixes les axes, et tous les changements obtenus dans les racines de l'équation, n'affectent en aucune manière la forme même de son lieu géométrique. - Mais, puisque l'on peut attribuer à chacun des coefficients de l'équation une infinité de valeurs différentes depuis $-\frac{1}{0}$ jusqu'à $+\frac{1}{0}$, on pourra, sans changer le degré de l'équation ni la position des axes, faire varier la courbe même d'une infinité de manières, sans que celle courbe change de nature, ou cesse de présenter les phénomènes généraux de courbure, qui doivent y caractériser le degré de l'équation.

Soit par exemple (fig. 8) la courbe $\boldsymbol{D} r m m^{\prime} \boldsymbol{A}$, qui représente le lieu géométrique d'une équation du $3^{\text {me }}$ degré, dont une seule racine est réelle; si l'on fait varier les coefficients de cette équation, on fera varier la distance entre le point de maximum $m$, et le point de minimum $m^{\prime}$, et parconséquent la longueur de la portion de courbe qui sépare ces deux points; - mais il est évident que parmi loutes les suppositions que l'on peut faire, il doit nécessairement s'en trouver de telles, que celte portion de courbe devienne zéro, ou, en d'autres termes, que le point de maximum $m$ et le point de minimum $\boldsymbol{n}^{\prime}$, viennent à se confondre en un seul et même point d'inflexion, comme l'indique la courbe ponctuée: $D^{\prime} r^{\prime} m^{\prime \prime} A^{\prime}$, dont la tangente $\boldsymbol{T}^{\prime \prime} \boldsymbol{T}^{\prime \prime}$ au point d'inflexion, est parallèle à l'axe des $x$.

D'autres valeurs des coefficients de l'équation, pourraient réduire à zéro une portion de courbe nmm'n', plus grande que $m m^{\prime}$, et la courbe prendrail alors la figure indiquée par la ligne poncluée $D^{\prime \prime} r^{\prime \prime} m^{\prime \prime} A^{\prime \prime}$, et les deux 
points $n$ et $n^{\prime}$ se réuniraient en un seul point d'inflexion $m^{\prime \prime \prime}$, dont la tangente $\boldsymbol{T}^{\prime \prime \prime} \boldsymbol{T}{ }^{\prime \prime \prime}$ est inclinée à l'axe des $x$. Dans l'un et l'autre cas, ce point d'inflexion indique deux racines imaginaires s'il est situé hors de l'axe des $x$, comme dans la (fig. 8). - Au contraire si ce point d'inflexion est sur l'axe mème des $x$, alors, il correspond à trois racines égales dans le cas où l'axe est tangent à la courbe, et à une racine réelle et deux racines imaginaires, si la tangente en ce point est inclinée sur l'axe des $x$.

En effet, l'équation du $3^{\text {me }}$ degré qui aurait toutes ses racines égales à ta par exemple, pourrait être mise sous la forme

$$
(x-a)^{3}=0 \text {, }
$$

et l'on voit que cette équation et ses deux premières dérivées deviennent zéro quand on $\mathrm{y}$ fait $x=+a$, ce qui prouve que la courbe a sur l'axe des $x$ un point d'inflexion dont l'abscisse est $+a$, et qui a cet axe même pour tangente.

L'équation du $3^{\text {me }}$ degré qui peut se mettre sous la forme:

$$
(x-a)\left((x-a)^{2}+b^{2}\right)=0,
$$

ou bien:

$$
(x-a)^{3}+(x-a) b^{2}=0,
$$

n'a qu'une racine réelle $a$, qui satisfait à cette équation et à sa seconde dérivée, mais celle valeur réduit à $+b^{2}$ la première dérivée, ce qui prouve que le point d'inflexion a pour tangente une droite inclinée à l'axe de $x$, et qui fait avec cet axe un angle, dont la tangente trigonométrique est $f-b^{2}$.

Cet exemple suffí pour faire comprendre le genre de variations que peuvent éprouver les lieux géométriques des équations; - ainsi par exemple il peut arriver que deux points de maximum et le point de minimum intermédiaire, ou deux points de minimum et un de maximum, se réunissent en un seul point, et dans un tel cas le point qui en résulte pourra correspondre: soit à quatre racines égales, soit à deux racines égales et à deux racines imaginaires, si l'axe touche la courbe en ce point; - soit à deux, ou à quatre racines imaginaires, si le point n'est pas sur l'axe des $x$.

Les points singuliers d'un lieu géométrique, formés par la réunion de deux ou de plusieurs points singuliers en un seul, peuvent être désignés sous le nom de points singuliers composés, pour les distinguer des points singuliers que la courbe présente dans le cas général, et que nous nommerons points singuliers simples ou ordinaires. 
Nous dirons donc en résumé: qu'un point de maximum ou de minimum simple, correspond à deux racines égales chaque fois que l'axe de $x$ touche la courbe en ce point, et, à deux racines imaginaires, quand la courbe tourne sa convexité vers l'axe des $x$ sans le couper ni le toucher.

Qu'un point de maximum ou de minimum composé, quand la courbe touche l'axe en ce point, correspond à plusieurs couples de racines égales, ou, à un couple au moins de racines égales, et un, ou plusieurs couples de racines imaginaires. - Que quand un tel point tourne sa convexilé vers l'axe sans le toucher, il correspond à autant de couples de racines imaginaires, qu'il réunit en lui de points de maximum ou de minimum simples, qui auraient leur convexilé tournée dans le même sens; - enfin, que quand un tel point est situé hors de l'axe de $x$, et que la courbe en ce point tourne sa concavité vers cet axe: ce point correspond à autant de couples, moins un, de racines imaginaires, qu'il réunit en lui de points de maximum et de minimum simples, dont la convexité serait tournée dans le même sens.

Un point d'inflexion simple, ou ordinaire, ne correspond à une racine que quand ce point tombe par hazard sur l'axe des $x$; hors de cet axe, ce point ne correspond à aucune racine réelle ni imaginaire.

Un point d'inflexion composé, quand il est sur l'axe des $x$, correspond a un nombre toujours impair de racines égales et un ou plusieurs couples de racines imaginaires, si l'axe est tangent à la courbe en ce point, et à une seule racine réelle et un ou plusieurs couples de racines imaginaires, si la tangente est inclinée à l'axe des $x$. - Lorsque le point d'inflexion composé se trouve hors de l'axe des $x$, il correspond toujours à un ou plusieurs couples de racines imaginaires.

Il faut observer que les points d'inflexion composés, ont une position inverse de celle des points d'inflexion simples; - ainsi, que l'on mène par un point d'inflexion simple deux droiles parallèles aux axes des coordonnées, on verra que les deux portions de courbe qu'il sépare auront leur concavité tournée vers la ligne qui est parallèle à l'axe des $x$, et leur convexilé vers celle qui est parallèle à l'axe des $y$, tandis que le contraire aura lieu pour un point d'inflexion composé.

Soit

$$
\boldsymbol{X}=(\boldsymbol{x}-\boldsymbol{a})^{2} \mathfrak{X}=\mathbf{0}
$$

une équation qui a deux racines égales à $+a ;-\mathfrak{X}$ étant un polynome indépendant du facteur $(x-a)$. - On aura en différentiánt 
15. F. Theremin, sur la résolution des équations.

$$
\begin{aligned}
& \boldsymbol{X}^{\prime}=2(x-a) \mathfrak{X}+(x-a)^{2} \mathfrak{X}^{\prime} \\
& \boldsymbol{X}^{\prime \prime}=2 \mathfrak{X}+2(x-a) \mathfrak{X}^{\prime}+(x-a)^{2} \mathfrak{X}^{\prime \prime}
\end{aligned}
$$

$\mathfrak{X}^{\prime}$ et $\mathfrak{X}^{\prime \prime}$ étant les dérivées du polynôme $\mathfrak{X}$.

En examinant ces trois équations, on voit, que $(x-a)$ est deux fois facteur de $\boldsymbol{X}$, une fois seulement de $\boldsymbol{X}^{\prime}$ et qu'enfin ce facteur ne divise plus $\boldsymbol{X}^{\prime \prime}$, d'où il suit que la première dérivée aura une racine égale à $+a$, ou à la racine double de l'équation donnée.

Il résulte de cela, qu'en déterminant successivement les racines des équations dérivées, en commençant par celles qui sont du $1^{\text {er }}$ et du second degré, et en substituant les valeurs trouvées pour une dérivée, dans l'équation d'un indice immédiatement supérieur pour déterminer les points de maximum et de minimum de cette équation, il arrivera que si l'une des racines a de l'une des dérivées de l'indice $n$, satisfait à la dérivée de l'indice immédiatement supérieur $(n-1)$, on doit en conclure que celte dernière équation a une racine double.

Mais, il se présente ici une difficulté, qu'il est indispensable de lever. Voici en quoi elle consiste: - si l'on pouvait déterminer exactement dans chaque cas semblable la racine $+a$, il est évident, que dans le cas de deux racines égales, l'équation de l'indice $(n-1)$ serait aussi exactement satisfaite, et. il ne saurait alors y avoir aucun doute; - mais, comme la quantité $a$ peut être incommensurable, et que dans ce cas on ne peut jamais avoir sa valeur exacte, et ensuite, que même quand la racine $a$ est une quantité finie il faut souvent pousser l'approximation très loin pour la découvrir, il doit nécessairement arriver qu'en se contentant d'une approximation de deux, trois, même de quatre chiffres décimaux exacts, la valeur de $a$ ainsi déterminée et subslituée dans l'équation de l'indice $(n-1)$, ne réduira pas cette équation exactement à zéro, mais donnera une valeur, positive ou négative extrêmement petite pour l'ordonnée du point de maximum ou de minimum correspondant à la racine cherchée; - parconséquent on ne peut pas être assuré directement dans ce cas, si en ce point l'équation a effectivement deux racines égales, ou deux racines imaginaires, ou enfin deux racines réelles mais très peu différentes entr'elles. - Il est donc nécessaire d'indiquer le moyen de s'en assurer exactement

Soit (fig. 9) une portion $c ı n c^{\prime}$ du lieu géométrique de l'équation quelconque $\boldsymbol{X}=0$, qui correspond au point pour lequel la quantité $x=u$, racine 
approchée de $\boldsymbol{X}^{\prime}=0$, donne une valeur très petite pour $\boldsymbol{X}$. Les trois cas que nous avons à éxaminer sont représentés par la (fig. 9) en $\boldsymbol{A}$, $\boldsymbol{B}$ et $\boldsymbol{C}$.

Soit $O n=a$, la valeur approchée de la racine de $X^{\prime}$, moindre que cette racine, et qui, substituée dans le polynome $X$, donne une valeur extrêmement petite $+n p$ pour l'ordonné. - Il est évident que si la courbe touche ou coupe l'axe des $x$, quelque loin que l'on pousse l'approximation, on ne pourra jamais être certain qu'il y ait, soit un point de contact, soit deux points d'intersection extrêmement voisins; - dans un tel cas, après avoir poussé l'approximation jusqu'à trois ou quatre chiffres décimaux exacts, on formera une seconde valeur approchée de la racine, en augmentant d'une unité le dernier chiffre décimal exact de la valeur trouvée, et celte nouvelle valeur approchée que nous nommerons $b$, si elle ne satisfait pas l'équation, sera évidemment plus grande que la racine, qui se trouvera parconséquent comprise entre $a$ et $b$.

Dans le cas où la courbe coupe l'axe des $x$, comme on le voit dans la (fig. 9) en $(A)$, il arrivera de deux choses l'une: $1^{\circ}$. Ou la valeur de $b$ mise dans le polynome $\boldsymbol{X}$ donnera une ordonnée négative très petite, et alors on sera certain que la courbe coupe l'axe entre $a$ et $b$ et qu'elle a parconséquent deux racines réelles, inégales, quoique très voisines. $-2^{\circ}$. Ou la valeur de $b$ mise dans l'équation donnera une ordonnée $n^{\prime} p^{\prime}$, très petite aussi, mais positive, comme on le voit en $(\boldsymbol{A}),(\boldsymbol{B})$ et $(\boldsymbol{C})$.

Dans le premier cas, on pourra considérer la valeur approchée $a$, comme une valeur suffisamment approchée de l'une des racines, et l'on cherchera l'autre par le procédé ordinaire en partant de l'abscisse du point d'inflexion voisin; - dans le second cas, il faudra prendre $a$ et $b$ pour points de départ de nouvelles approximations, et, ayant chaque fois formé les valeurs des soustangentes $n s$ et $n^{\prime} s^{\prime}$, les ajouter ensemble abstraction faite du signe, pour voir si leur somme atteint ou dépasse la différence $b-a$ ou $n n^{\prime}$ des deux valeurs dont on est parti. - En effet, si les deux racines sont réelles, il suffit de voir les fig. $(A)$ et $(B)$ pour se convaincre que la somme des deux soustangentes ne peut jamais atteindre la différence $b-a$; - mais an contraire, si les deux racines sont imaginaires comme dans la fig. $(C)$, la courbe ne coupera pas l'axe des $x$, et il est évident qu'il suffira d'un très petit nombre d'approximations pour que la somme des soustangentes dépasse $b-a$. 
$a$ et $b$ élant les valeurs des deux abscisses, l'une $a$ moindre que la racine, l'autre $b$ plus grande, nous aurons:

$$
\frac{\frac{X_{(a)}}{X_{(a)}^{\prime}}+\frac{X_{(b)}}{X_{(b)}^{\prime}}}{b-a}
$$

pour le rapport de la somme des soustangentes à la différence des limites $a$ et $b$ dont on était parti, et, en continuant l'approximation, on aura:

$$
\frac{\frac{X_{\left(a^{\prime}\right)}}{X_{\left(a^{\prime}\right)}^{\prime}}+\frac{X_{\left(b^{\prime}\right)}}{X_{\left(b^{\prime}\right)}^{\prime}}}{b^{\prime}-a^{\prime}}
$$

pour le même rapport après une nouvelle approximation, et ainsi de suite. Mais il est évident: que si les racines sont imaginaires, ce rapport ira promptement en croissant, et dépassera bientôt l'unité; - que si les deux racines sont réelles et inégales, ce rapport au lieu de croître diminuera rapidement; enfin, que si les deux racines sont égales, ce rapport s'approchera de l'unité sans jamais la dépasser ni l'atteindre. - Dans le premier cas, aussitôt que le rapport sera devenu $>1$ on discontinuera l'approximation, et l'on sera certain que les deux racines manquent, ou sont imaginaires entre $a$ et $b$. Dans le second cas on verra dès la $2^{\text {de }}$ approximation que les deux racines sont réelles et inégales, et on pourra continuer à les approximer séparément, jusqu'à tel nombre de chiffres décimaux exacts qu'on voudra. - Enfin, dans le troisième cas, on se contentera d'approximer la racine de l'un ou de l'autre côté du point de contact, ou bien on cherchera le facteur commun de $\boldsymbol{X}$ et $\boldsymbol{X}_{1}$ par le procédé du plus grand commun diviseur, et ce facteur donnera la racine double exactement.

Une équation qui aurait un nombre $n$ de racines égales, serait de la forme:

$$
\boldsymbol{X}=(\boldsymbol{x}-\boldsymbol{a})^{n} \mathfrak{X}=\mathbf{0}
$$

et il est évident, que: la première dérivée de cette équation serait divisible par $(x-a)^{n-1}$, la seconde par $(x-a)^{n-2}$, la troisième par $(x-a)^{n-3}$, etc. jusqu'à la $(n-1)$ ième qui ne contiendrait plus le facteur $(x-a)$ qu'au premier degré. - Cela prouve que quand une équation a un nombre $n$ de racines égales à $a$, sa première dérivée en aura $(n-1)$, sa seconde $(n-2)$ et ainsi de suite, et que parconséquent, cette équation et ses $(n-1)$ premières dérivées auront un facteur commun. - Il résulte de là, qu'en posant $\mathfrak{X}=1$, l'équation aura toutes ses racines égales, et toutes ses dérivées, hors la dernière seront 
divisibles par $x$ - $a$, et parconséquent la racine multiple $a$, serait donnée par l'avant dernière dérivée qui ne contient le facteur $(x-a)$ qu'au $1^{\text {er }}$ degré.

Les racines imaginaires, hors le cas que nous avons examiné (c'est à dire celui où la courbe passe très près de l'axe), ne présent aucune difficulté, puisqu'en déterminant successivement les racines de chaque dérivée, en commençant par celles du $1^{\text {er }}$ et du second degré, on détermine au moyen de ces racines, la position des points de maximum, de minimum, et d'inflexion, de la dérivée d'un degré immédiatement supérieur, et la position de ces points indique de suite si le lieu géométrique auquel ces points appartiennent coupe ou non l'axe des $x$ en autant de points que cette dérivée doit avoir en tout de racines selon son degré. - Les points d'intersection qui manqueront, seront autant de racines imaginaires, et correspondront toujours par couples, soit à un point de maximum ou de minimum, soit à un point d'inflexion composé.

On voit donc, qu'en commençant l'analyse d'une équation par l'avant dernière dérivée, on s'élève successivement à la connaissance des racines de toutes les dérivées de cette équation, et enfin à la connaissance des racines de la proposée, et que cette analyse fait connaitre la nature des racines cherchées, et la valeur de celles qui sont réelles avec tel degré d'approximation qu'on voudra.

Observation. Dans le cas où toutes les racines sont réelles et inégales, nous avons vu qu'on obtenait les racines extrêmes par la formation d'une équation du $2^{\mathrm{d}}$ degré; dans le cas où il $\mathrm{y}$ a des racines imaginaires ce procédé n'est plus applicable, et l'on déterminera les racines extrêmes directement, par approximation, en prenant la quantité arbitraire $\alpha$, plus grande que l'abscisse du dernier minimum du côté positif, pour la limite positive, et, moindre que l'abscisse positive (ou plus grande que l'abscisse négative) du dernier point de minimum ou de maximum du côté négatif, pour la limite négative. - On sera sur qu'en choisissant ainsi ces quantités arbitraires, il n'y aura aucun point singuliers entr'elles et la racine cherchée, et parconséquent l'approximation sera convergente dès le second terme, si $\alpha$ est moindre que la racine cherchée, et dès le $1^{\text {er }}$ si cette quantité dépasse la racine.

\section{IX.}

Après avoir développé dans les articles précédents, la marche à suivre pour découvrir successivement toutes les racines d'une équation quel que soit son degré, il convient d'examiner la convergence que donne la formule, et 
les moyens d'évaluer le degré de chacunes des approximations obtenues par l'emploi de la méthode proposée, et d'indiquer le moyen d'arriver promptement à la détermination de la quantité arbitraire $\alpha$ la plus convenable au point de départ de chaque opération.

Soit Armr'im' une portion de courbe, (fig. 10), qui coupe l'axe des $x$ en deux points $r$ et $r^{\prime}$, et dont les points de maximum et de minimum $m^{\prime}$ et $\boldsymbol{m}$ sont déjà déterminés par les opérations précédentes; - supposons aussi que in $\boldsymbol{r} \boldsymbol{A}$, 'soit l'une des branches extrêmes de la courbe; - il s'agit de déterminer les deux racines $\boldsymbol{O} \boldsymbol{r}$ et $\boldsymbol{O} \boldsymbol{r}^{\prime}$ qui correspondent à cette portion de la courbe.

Pour la première de ces racines, $O r$, nous n'avons pas d'autres données si non que la quantité arbitraire $\alpha$, qui doit servir de point de départ à l'approximation, doit être moindre que l'abscisse $O a$ du point minimum $m$, mais en même temps -il est évident que moins la quantité prise pour point de départ différera de la racine cherchée, et plus l'approximation sera rapide; - or, pour atteindre ce but, on substituera dans le polynôme $\boldsymbol{X}$ le plus grand nombre entier $\boldsymbol{O} a^{\prime}$, qui soit contenu dans l'abscisse $\boldsymbol{O} a$; puis on diminuera ce nombre de l'unité et on le substituera de nouveau dans le polynôme $\boldsymbol{X}$ au lieu de $\boldsymbol{x}$, et ainsi de suite, et, à chaque substitution nouvelle on examinera le signe du résultat, et l'on continuera de la même manière jusqu'à ce que le résultat de la substitution change de signe. - De cette manière on parvient facilement à deux abscisses: $O a^{\prime \prime}$ et $O a^{\prime \prime \prime}$, dont l'une $O a^{\prime \prime}$ est plus grande que la racine $O r$, et l'autre $O a^{\prime \prime \prime}$ qui est moindre, et qui ne diffère de la précédente que d'une seule unité. - Si nous nommons $\alpha$ le plus petit et $\beta$ le plus grand de ces deux nombres, nous aurons donc: $\beta-\alpha=1$, parconséquent $\alpha$ est le plus grand nombre entier contenu dans la racine cherchée, et comme tel, nous pouvons prendre ce nombre pour bâse de l'approximation.

Pour la racine $O \boldsymbol{r}^{\prime}$, qui se trouve entre le point d'inflexion $i$ et le point de minimum, $m$, si la portion $a b$ de laxe, qui se trouve entre ces deux points contient un certain nombre d'unités, on prendra d'abord le plus grand nombre entier contenu dans l'abscisse $O b$ du point d'inflexion, et on substituera ce nombre à $x$, dans le polynôme $X$, pour voir s'il change de signe; si ce nombre ne change pas le signe du polynôme $\boldsymbol{X}$, on le prendra pour bâse d'une première approximation, qui donnera une première valeur $\alpha^{\prime}$; on prendra ensuite le plus grand nombre entier contenu dans $\alpha^{\prime}$, pour bâse d'une second approximation, et l'on continuera de la sorte, en n'opérant que sur des nombres entiers, jusqu'à ce qu'on arrive à un nombre entier qui fasse 
changer le signe de $\boldsymbol{X}_{\text {; }}$ alors on sera certain que la racine est comprise entre le dernier nombre déduit par la formule d'approximation, et le plus grand nombre entier qu'il contienne.

Si la racine cherchée était située sur une portion de courbe tournant sa convexité vers l'origine, alors on prendrait le nombre entier le plus petit qui puisse contenir l'abscisse du point d'inflexion; - puis après avoir trouvé au moyen de ce nombre la première approximation $\alpha^{\prime}$, on prendrait le plus petit nombre entier qui puisse contenir $\alpha^{\prime}$, et ainsi de suite, jusqu'à ce qu'on arrive à un nombre entier qui fasse changer le signe de $X_{\text {; }}$ alors, on est certain que la racine est comprise entre ce dernier nombre entier et le résultat précédent.

On voit donc que par ce procédé, et en opérant seulement sur des nombres entiers, on parvient au bout d'un petit nombre d'opérations fort simples, $\dot{a}$ trouver deux nombres $\alpha$ et $\beta$, qui ne diffèrent de la racine cherchée, l'un en plus, l'autre en moins, que d'une quantité moindre que l'unité, et que parconséquent il faut prendre l'un de ces nombres pour bâse de l'approximation dans le but de découvrir la partie décimale de la racine.

Examinons à présent, quelle peut être la rapidité de l'approximation que donne la formule, et cherchons à découvrir l'ordre d'unités décimales auquel appartient chacun des résultats successifs de l'opération.

Soit $O m$ (fig. 11) l'abscisse $\alpha$ qui sert de point de départ à l'approximation, et qui est telle, que la différence entre $\alpha$ et la racine, ou entre $O_{m}$ et $O r$ est moindre que l'unité. - Nous avons pour première approximation:

$$
\alpha^{\prime}=\alpha-\frac{X_{(\alpha)}}{X_{(\alpha)}^{\prime}}=\boldsymbol{O} m+m t,
$$

ou en posant $m t=i$,

$$
\alpha^{\prime}=\alpha+i^{\prime}, \quad \text { et } \quad+i=-\frac{X_{(\alpha)}}{X_{\alpha}^{\prime}} .
$$

Pour seconde approximation, nous aurons:

ou en posant $t t^{\prime}=i^{\prime}$,

$$
\alpha^{\prime \prime}=\alpha^{\prime}-\frac{X_{\left(\alpha^{\prime}\right)}}{X_{\left(\alpha^{\prime}\right)}^{\prime}}=0 t+t^{\prime},
$$

$$
\alpha^{\prime \prime}=\alpha^{\prime}+i^{\prime}=\alpha+i+i^{\prime}, \quad \text { et } \quad i^{\prime}=-\frac{X_{\left(\alpha^{\prime}\right)}}{X_{\left(\alpha^{\prime}\right)}^{\prime}} \text {. }
$$

Cherchons la relation qui existe entre $i$ et $i^{\prime}$; - pour cela, menons par le point $p^{\prime}$ la ligne $p^{\prime} \varphi$ parallèle à la tangente $t$, on aura évidemment: 
15. F. Theremin, sur la résolution des équations.

d'oụ l'on tíre:

$$
p m: m t=p^{\prime} t: t q, \quad \text { ou } \quad X_{(\alpha)}: i=X_{\left(\alpha^{\prime}\right)}: t q,
$$

$$
t q=i \frac{X_{\left(\alpha^{\prime}\right)}}{X_{(\alpha)}}
$$

mais, puisque $i^{\prime}$ ou $\| t^{\prime}$ est une fraction fort petite, on voit qu'en négligeant des quantités fort petites du $2^{\mathrm{d}}$ degré, on peut considérer comme égales les lignes tq et $q t^{\prime}$; et poser

d'où il résulte:

$$
t q=q t^{\prime}=\frac{1}{2} t t^{\prime}=\frac{1}{2} i^{\prime}
$$

mais puisque $\alpha^{\prime}=\alpha+i$, on aura:

$$
i^{\prime}=2 i \frac{X_{\left(\alpha^{\prime}\right)}}{X_{(\alpha)}}
$$

$$
\boldsymbol{X}_{\left(\alpha^{\prime}\right)}=\boldsymbol{X}_{(\alpha+i)}=\boldsymbol{X}_{(\alpha)}+i \boldsymbol{X}_{(\alpha)}^{\prime}+\frac{1}{2} i^{2} \boldsymbol{X}_{(\alpha)}^{\prime \prime}+\cdots
$$

En se bornant aux trois premiers termes de ce développement, et en les substituant dans l'expression de $i^{\prime}$, on trouve:

$$
i^{\prime}=\frac{2 i X_{(\alpha)}+2 i^{2} X_{(\alpha)}^{\prime}+i^{3} X_{(\alpha)}^{\prime \prime}}{X_{(\alpha)}}=2 i\left\{1+i \frac{X_{(\alpha)}^{\prime}}{X_{(\alpha)}}\right\}+i^{3} \frac{X_{(\alpha)}^{\prime \prime}}{X_{(\alpha)}}
$$

Mais puisque: $+i=-\frac{X_{(\alpha)}}{X_{(\alpha)}^{\prime}}$, il s'ensuit que la quantité qui est entre parenthèses devient nulle, et il ne reste que: $i^{\prime}=+i^{3} \frac{X_{(\alpha)}^{\prime \prime}}{X_{(\alpha)}}$, ou bien, en remplaçant $\boldsymbol{X}_{(\alpha)}$ par $-i \boldsymbol{X}_{(\alpha)}^{\prime}$, et simplifiant:

$$
\boldsymbol{i}^{\prime}=-\boldsymbol{i}^{2} \frac{\boldsymbol{X}_{(\alpha)}^{\prime \prime}}{\boldsymbol{X}_{(\alpha)}^{\prime}}
$$

On voit donc, que la quantité $i^{\prime}$ dont le résultat de l'approximation s'accroit à chaque opération, est proportionnel: $1^{\circ}$, à la second puissance de la quantilé $i$, dont l'opération précédente avait augmenté la bâse $\alpha$ dont on était parti, et $2^{\circ}$, à la quantite $\frac{X_{(\alpha)}^{\prime \prime}}{X_{(\alpha)}^{\prime}}$; il doit être bien entendu d'ailleurs, que cette loi n'est vraie qu'en supposant qui $i$ est une fraction assez petite.

Supposons d'abord, que $\frac{X_{(\alpha)}^{\prime \prime}}{X_{(\alpha)}^{\prime}}$ abstraction faite du signe, soit égal à l'unité, et que l'on soit parvenu à une approximation de la racine que nous représenterons par: $e, a b c$, exprimant par $e$ le nombre et par $a, b$, et $c$, les trois premiers chiffres décimaux exacts; - si nous posons: $\alpha=e, a b$ et $\alpha^{\prime}=e, a b c$, nous aurons $i=0,00 c$, parconséquent:

$$
\alpha^{\prime \prime}=\alpha^{\prime}+i^{\prime}=\alpha+i+i^{2}=e, a b c+(0,00 c)^{2}=e, a b c 00 c^{2} .
$$


Mais, $c^{2}$ ne pouvant être qu'un nombre de deux chiffres au plus, on voit que la seconde approximation, ne peut affecter que les caractères décimaux du cinquième et du sixième rang, et que parconséquent les premiers quatre chiffres décimaux, au moins, sont exacts; - en nommant $d$ le quatrième chiffre, nous aurons donc: e, abcd pour résultat; - mais en posant de nouveau : $\alpha=e, a b c$, et $i=0,000 d$, nous aurons:

$$
\alpha^{\prime \prime}=\alpha+i+i^{2}=e, a b c d+(0,000 d)^{2}=e, a b c d 000 d^{2} .
$$

Ce qui fait voir que celte nouvelle opération ne peut affecter que le $7^{\text {mee }}$ et le $8^{\text {me }}$ chiffre, et que parconséquent les six chiffres du résultal précédent étaient tous exacts. - Il suit de là, que quand on a l'expression de la racine avec trois chiffres décjmaux exacts, l'opération suivante en donnera six.

Soit donc: e,abcdef l'expression de la racine avec six chiffres exacts; posons: $\alpha=e, a b c d e$ et $i=0,00000 f$, nous aurons comme ci-dessus:

$$
\alpha^{\prime \prime}=\alpha+i+i^{2}=e, a b c d e f+0,00000000000 f^{2}=e, a b c d e f 00000 f^{2} .
$$

Ce qui prouve que quand on a cinq chiffres décimaux exacts, l'opération suivante en donne dix, et l'on voit qu'en poursuivant de cette manière on démontrerait aisément qu'à chaque opération nouvelle le nombre des décimales exactes se trouve doublé.

Si la quantité $\frac{X_{(\alpha)}^{\prime \prime}}{X_{(\alpha)}^{\prime}}$ au lieu d'être égale à l'unilé comme nous lavions supposée, était moindre, il est évident que la rapidité de l'approximation serait encore plus grande, mais au contraire cette rapidité se trouverait diminuée, et le nombre des chiffres décimaux exacts de chaque opération serait moindre, si $\frac{X_{(\alpha)}^{\prime \prime}}{X_{(\alpha)}^{\prime}}$ était plus grand que l'unilé.

Observons, que quand la racine cherchée sera proche d'un point d'inflexion du lieu géométrique de $\boldsymbol{X}$, la quantité $\boldsymbol{X}_{(\alpha)}^{\prime \prime}$ deviendra fort petite, puisque l'abscisse du point d'inflexion de $\boldsymbol{X}$ est la même que celle qui correspond à la racine de $\boldsymbol{X}^{\prime \prime}$; - en même temps, la quantité $\boldsymbol{X}_{(\alpha)}^{\prime}$ devient la plus grande possible, puisque la même abscisse correspond à celle d'un point de maximum ou de minimum de $\boldsymbol{X}^{\prime}$; - parconséquent dans ce cas, le facteur $\frac{X_{(\alpha)}^{\prime \prime}}{\boldsymbol{X}_{(\alpha)}^{\prime}}$ devient le plus petit possible. - Le contraire a lieu, quand la racine cherchée est peu éloignée d'un point de maximum ou de minimum da lieu géométrique 
de $\boldsymbol{X}$, car alors c'est $\boldsymbol{X}_{(\alpha)}^{\prime}$ qui diminue et s'approche de zéro, et parconséquent $\frac{X_{(\alpha)}^{\prime \prime}}{\boldsymbol{X}_{(\alpha)}^{\prime}}$ peut devenir une quantité très grande.

Celte observation n'ôte rien à la valeur du mode d'approximation adopté, mais comme il peut en résulter de l'incertilude sur le nombre des chiffres décimaux exacts de chaque opération, il faut écarter celte incertitude soit en déterminant la quantité $\frac{X_{(\alpha)}^{\prime \prime}}{X_{(\alpha)}^{\prime}}$, soit en employant comme Fourrier, deux limites.

Supposons, pour cela, (fig. 12) que l'on soit arrivé par les opérations précédentes à deux abscisses $O m=\alpha$ et $O m^{\prime}=\beta$, dont la première est moindre et la seconde plus grande que la racine $O r$ qu'il faut déterminer, et qui ne diffèrent entr'elles que de l'unité; - la courbe tournant sa convexité vers le point $m$, il faudra commencer l'approximation par la plus pelite des deux limites.

Élévons les ordonnées $m p$ et $m n^{\prime} p^{\prime}$, et menons au point $p$ la tangente $t t$ $\dot{a}$ la courbe, nous aurons construit la première approximation:

$$
\boldsymbol{O} t=\boldsymbol{O} m+\boldsymbol{m} t=\alpha-\frac{X_{(\alpha)}}{X_{(\alpha)}^{\prime}}=\alpha^{\prime}
$$

qui est moindre que la racine cherchée. - Ensuite, par le point $\boldsymbol{p}^{\prime}$ menons la ligne $p^{\prime} q$ parallèle à la tangente $t$, cette ligne coupera l'axe en un point $q$ situé entre $\boldsymbol{r}$ et la plus grande limite $\beta$. - Mais, puisque les triangles: mpt et $m^{\prime} p^{\prime} y$ sont semblables, et que l'ordonnée $m^{\prime} p^{\prime}$ a pour expression $\boldsymbol{X}_{(\beta)}$, nous aurons évidemment:

et parconséquent:

$$
\boldsymbol{n}^{\prime} \boldsymbol{\varphi}=\frac{\boldsymbol{X}_{(\beta)}}{\boldsymbol{X}_{(\alpha)}^{\prime}}
$$

$$
\boldsymbol{O} q=0 m^{\prime}-m^{\prime} q=\beta-\frac{X_{(\beta)}}{X_{(\alpha)}^{\prime}}=\beta^{\prime} .
$$

Mais il est évident que les quantités $\alpha^{\prime}$ et $\beta^{\prime}$ ainsi construites, sont deux nouvelles limites, l'une, $\beta^{\prime}$, plus grande, et l'autre, $\alpha^{\prime}$, moindre que la racine cherchée, quoique beaucoup plus rapprochées d'elle que ne l'étaient les nombres $\alpha$ et $\beta$ dont on était parti. - Pour avoir des limites encore plus rapprochées, répetons sur $\alpha^{\prime}$ et $\beta^{\prime}$ la même opération, nous aurons:

$$
\alpha^{\prime}-\frac{X_{\left(\alpha^{\prime}\right)}}{X_{\left(\alpha^{\prime}\right)}^{\prime}}=\alpha^{\prime \prime} \quad \text { et } \quad \beta^{\prime}-\frac{X_{\left(\beta^{\prime}\right)}}{X_{\left(\alpha^{\prime}\right)}^{\prime}}=\beta^{\prime \prime},
$$

et ainsi de suite, et à chaque opération nous obtiendrons deux nouvelles limites

Crelle's Journal f. d. M. Bd. XLIX. Heft 3. 
qui se rapprocheront continuellement et finiront par ne plus différer entr'elles que par les chiffres décimaux d'un rang très éloigné.

Supposons par exemple qu'à la troisième opération on ait trouvé :

$$
\begin{aligned}
& \alpha^{\prime \prime \prime}=e, \text { abcdef.g ...., } \\
& \beta^{\prime \prime \prime}=e, \text { abcdelm...., }
\end{aligned}
$$

puisque la racine est comprise entre ces deux nombres, il est évident que tous les chiffres: e, abcde qui sont les mêmes dans les deux limiles, appartiennent à la racine cherchée, et que parconséquent la dernière opération détermine cinq chiffres décimaux exacts.

Pour compléter l'exposé de la méthode, il ne reste plus que d'en faire quelques applications, ce qui sera l'objet des articles suivants.

\section{$\mathbf{X}$}

Après avoir exposé d'une manière générale la méthode pour la détermination des racines des équations de tous les degrés, avec tel degré d'approximation qu'on voudra, faisons quelques applications qui en faciliteront l'intelligence.

Pour premier exemple, cherchons les racines de l'équation du $7^{\text {me }}$ degré

$$
X=+x^{7}+9 x^{6}-108 x^{5}+386 x^{4}-713 x^{3}+747 x^{2}-420 x+98=0,
$$
dont les dérivées seront:

$$
\begin{aligned}
& \boldsymbol{X}^{\prime}=+7 x^{6}+54 x^{5}-540 x^{4}+1544 x^{3}-2139 x^{2}+1494 x-420 \\
& \boldsymbol{X}^{\prime \prime}=+42 x^{5}+270 x^{4}-2160 x^{3}+4632 x^{2}-4278 x+1494, \\
& \boldsymbol{X}^{\prime \prime \prime}=+210 x^{4}+1080 x^{3}-6480 x^{2}+9264 x-4278 \\
& \boldsymbol{X}^{\mathrm{IV}}=+840 x^{3}+3240 x^{2}-12960 x+9264 \\
& \boldsymbol{X}^{\mathrm{v}}=+2520 x^{2}+6480 x-12960 \\
& \boldsymbol{X}^{\mathrm{vI}}=+5040 x+6480 \\
& \boldsymbol{X}^{\mathrm{vII}}=+5040 .
\end{aligned}
$$

D'abord, en égalant à zéro le polynome $\boldsymbol{X}^{\mathbf{v i}}$ qui est du premier degré, on trouve: $x=-1,28$ qui est l'abscisse du point de minimum, de la courbe que représente l'équation du second degré $X^{\mathrm{v}}=0$, ou $2520 x^{2}+6480 x-12960=0$, et aussi l'abscisse du point d'inflexion du lieu géométrique de $\boldsymbol{X}^{\mathrm{IV}}=0$.

Ensuite en résolvant l'équation $\boldsymbol{X}^{\mathrm{v}}=0$, on trouve deux racines réelles: $x=+0,80$ et $x=-3,36$, qui sont aussi les abscisses: du point de minimum et de celui de maximum de $\boldsymbol{X}^{\mathrm{Iv}}$. - Nous avons donc pour les points singuliers de $\boldsymbol{X}^{\mathrm{IV}}$ : 
13. F. Theremin, sur la résolution des équations.

Valeurs

des abscisses.
Désignation des points singuliers.
Valeurs des ordonnées correspondantes.

$-3,36 \ldots$ point de maximum $\ldots .+57.637,20 \ldots$

$-1,28 \ldots$ point d'inflexion simple ... . $\ldots+30.239,16 \ldots$

$+0,80 \ldots$ point de minimum $\ldots . .+1399,68 \ldots$

Puisque l'ordonnée du point de minimum est positive, il s'ensuit que la courbe aura la forme indiquée par la (fig. 13); - cette courbe sera située au dessus de l'axe $\left(-\boldsymbol{X}^{\mathrm{Iv}},+\boldsymbol{X}^{\mathrm{Iv}}\right)$ et aura parconséquent deux racines imaginaires, et une seule racine réelle, formée par l'intersection de l'axe $\left(-\boldsymbol{X}^{\mathrm{IV}},+\boldsymbol{X}^{\mathrm{IV}}\right)$ avec la branche gauche ou descendante de la courbe. - Pour trouver celte racine réelle, qui est située à gauche du point de maximum, dont l'abscisse est $-3,36$, il faut, dans $\boldsymbol{X}^{\mathrm{IV}}$ substituer des nombres entiers et négatifs plus grand que $-3,36$ jusqu'à ce que l'ordonnée change de signe; ainsi en substituant successivement $-4,-5,-6,-7$ etc. on trouvera:

$$
X_{(-6)}^{\mathrm{IV}}=+22224 \ldots \text { et } \boldsymbol{X}_{(-7)}^{\mathrm{IV}}=-29376 \ldots
$$

ce qui prouve que la racine cherchée est entre -6 et -7 .

L'abscisse -7 étant celle d'un point de l'axe qui est situé du côté de la convexité de la courbe, doit être prise pour bâse d'une première approximation, et l'on trouve:

et

$$
\boldsymbol{X}_{(-7)}^{\mathrm{iv}}=-29376
$$

d'où

$$
X_{(-7)}^{v}=+65150
$$

$$
x=-7-\frac{X_{(-7)}^{\mathrm{Iv}}}{X_{(-7)}^{\mathrm{v}}}=-7+\frac{29376}{65150}=-6,59 \ldots
$$

pour première approximation; - nous ne conserverons même, pour l'analyse de la dérivée supérieure $\boldsymbol{X}^{\prime \prime \prime}$, que le premier chiffre décimal de cette racine, et nous prendrons $-6,5$ pour l'abscisse correspondante à la racine de $X^{\text {iv }}$ et au point minimum de $\boldsymbol{X}^{\prime \prime \prime}$. -

Pour construire le lieu géométrique de l'équation $X^{\prime \prime \prime}=0$, qui est du $4^{\text {ine }}$ degré, nous avons donc:

Valeurs
des abscisses.

$$
\text { Points correspondants }
$$
de $X^{\text {IV }}$.

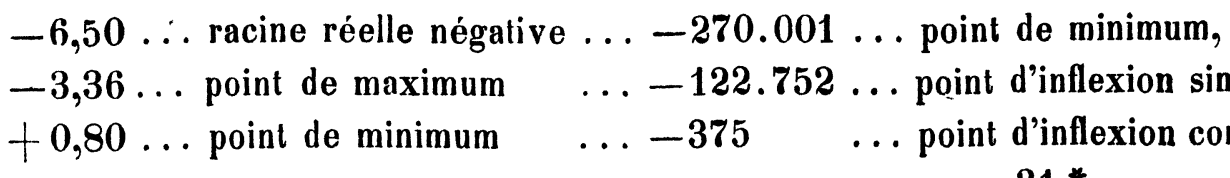

Ordonnées de $X^{\prime \prime \prime}$. Points correspondants
de $\boldsymbol{X}^{\prime \prime \prime}$. 
puisque les ordonnées de ces trois points sont toutes négatives, il s'ensuit que les racines réelles de $\boldsymbol{X}^{\prime \prime \prime}$ seront formées par l'intersection de l'axe $\left(-\boldsymbol{X}^{\prime \prime \prime},+\boldsymbol{X}^{\prime \prime \prime}\right)$ avec les deux branches extrêmes ou ascendante de la courbe; l'une de ces racines sera négative et plus grande que $-6,50$, c'est à dire que l'abscisse du point de minimum, et l'autre sera positive et plus grande que $+0,8$, ou que l'ạbscisse du point d'inflexion composée, qui, n'élant pas sur l'axe, indique un couple de racines imaginaires.

Pour déterminer la plus grande de ces deux racines, en mettant dans $\boldsymbol{X}^{\prime \prime \prime}$, successivement, les nombres: $-7,-8,-9,-10$, etc. nous trouvons:

$$
X_{(-9)}^{\prime \prime \prime}=-22404, \quad \text { et } \quad X_{(-10)}^{\prime \prime \prime}=+274.082 ;
$$

ce qui prouve que la racine est comprise entre les nombres -9 et -10 . En substituant ce dernier nombre dans la dérivée $\boldsymbol{X}^{\mathrm{rV}}$ on trouve:

$$
X_{(-10)}^{\mathrm{IV}}=-377.136
$$

ce qui donne les deux nouvelles limites:

$$
x=-10-\frac{X_{(-1)}^{\prime \prime \prime}}{X_{(-10)}^{\mathrm{v}}}=-10+\frac{274082}{377136}=-9,2 \ldots
$$

en nous bornant au $1^{\text {er }}$ chiffre décimal et

$$
x=-9-\frac{X_{(-9)}^{\prime \prime \prime}}{X_{(-1))}^{\mathrm{IV}}}=-9-\frac{22404}{377136}=-9,05
$$

pour limite inférieure.

La racine cherchée est donc comprise entre $-9,2$ et $-9,05$, et comme nous n'avons pas besoin ici d'une grande approximation, nous nous bornerons à prendre la moyenne entre ces deux limites, ce qui donne avec deux décimales le nombre $-9,12$ que nous porterons au tableau.

Pour la seconde racine, qui est plus grande que $+0,8$, nous voyons en mettant pour $x$ les nombres $+1,+2$, etc. que le nombre +2 fait changer de signe au polynôme $\boldsymbol{X}^{\prime \prime \prime}$, et que la racine cherchée est comprise, parconséquent, entre ces deux nombres. - Substituant donc aussi +2 dans le polynôme $\boldsymbol{X}^{\mathbf{i v}}$, et opérant comme ci-dessus, on aura deux limites plus rapprochées qui seront: $+1,80$ et $+1,06$ d'où l'on tire la moyenne $+1,43$, valeur approchée et que nous porterons au tableau. 
Nous aurons donc pour passer à $\boldsymbol{X}^{\prime \prime}$ :

Valeurs des abscisses.

\section{Points correspondants} de $\boldsymbol{X}^{\prime \prime \prime}$.
Ordonnées de $\boldsymbol{X}^{\prime \prime}$.
Points singuliers de $\boldsymbol{X}^{\prime \prime}$.

$-9,12 \ldots$

racine 1.282.034 .... point de maximum,

$-6,50 \ldots$ point de minimum $\ldots .+119.073 \ldots$ point d'inflexion simple, $+1,43 \ldots$ racine $\ldots-76$ .... point de minimum.

Puisque le lieu géométrique de $\boldsymbol{X}^{\prime \prime}$ n'a qu'un senl point de maximum au dessus de l'axe, et un seul point de minimum au dessous, il s'ensuit que ce lieu géométrique ne coupera l'axe qu'en trois points, et que parconséquent $\boldsymbol{X}^{\prime \prime}$ qui est du cinquième degré n'a que trois racines réelles. - L'une des racines, formée par l'intersection de l'axe avec la branche descendante, sera négative et plus grande que l'abscisse - 9,12 du point de maximum; - l'autre racine formée par l'intersection de l'axe avec la branche droite ou ascendante, sera positive et plus grande que l'abscisse $+\mathbf{1 , 4 3}$ du point de minimum; - enfin, la troisième racine sera formée par l'intersection de l'axe avec la portion de courbe comprise entre le point d'inflexion et le point de minimum, et sera comprise, parconséquent entre les valeurs $-6,50$ et $+1,43$ des abscisses de ces deux points.

Commençons par chercher cette dernière racine; - pour cela il faut substituer dans $X^{\prime \prime}$ successivement les nombres entiers compris entre $+1,43$ et $-6,50$ qui sont $+1,0,-1,-2,-3,-4,-5$ et -6 ; - or en commençant par le premier de ces nombres +1 , on trouve que $X^{\prime \prime}$ se réduit à zéro, et que parconséquent la racine cherchée est égale à +1 .

Pour la plus grande racine négative, mettons successivement les nombres $-10,-11,-12$, etc. dans le polynôme $X^{\prime \prime}$, nous trouvons:

$$
X_{(-11)}^{\prime \prime}=+572912 \text { et } X_{(-12)}^{\prime \prime}=-399.914 .
$$

Ce qui prouve que la racine cherchée est comprise entre les nombres -11 et -12 . - En mettant -12 , dans la première dérivée de $X^{\prime \prime}$, qui est $\boldsymbol{X}^{\prime \prime \prime}$, on trouve: $\boldsymbol{X}_{(-12)}^{\prime \prime \prime}=\frac{1}{+}$ 1.439.754, d'où l'on tire deux nouvelles limites:

$$
\left.\begin{array}{l}
x=-12-\frac{X_{(-12)}^{\prime \prime}}{X_{(-12)}^{\prime \prime}}=-12+\frac{399.914}{1.439 .754}=-11,7 \ldots \\
\text { et } \\
x=-11-\frac{X_{(-1)}^{\prime \prime}}{X_{(-12)}^{\prime \prime}}=-11-\frac{572.912}{1.439 .754}=-11,3 \ldots
\end{array}\right\} \begin{aligned}
& \text { en se bornant à la } \\
& \text { première décimale, }
\end{aligned}
$$


d'où l'on tire la moyenne $-11,5$ que nous porterons au tableau comme suffisamment approchée.

Enfin, pour la troisième racine, en substituant pour $x$ les nombres $+2,+3$, etc. plus grands que $+1,43$, l'on trouve:

$$
X_{(+2)}^{\prime \prime}=-150 \text { et } X_{(+3)}^{\prime \prime}=+4.104 \text {, }
$$

ce qui indique que la racine est entre +2 et +3 - En mettant +3 dans $\boldsymbol{X}^{\prime \prime \prime}$ on aura pour nouvelles limites:

et

$$
x=+3-\frac{X_{(+3)}^{\prime \prime}}{X_{(+3)}^{\prime \prime}}=+3-\frac{4104}{11364}=+2,6 \ldots
$$

$$
x=+2-\frac{X_{(+2)}^{\prime \prime}}{X_{(+3)}^{\prime \prime}}=+2+\frac{150}{11364}=+2,03 \ldots,
$$

d'où nous tirons la moyenne $+2,3$ que nous porterons au tableau.

\begin{tabular}{|c|c|c|c|}
\hline $\begin{array}{l}\text { Valeurs } \\
\text { des } \\
\text { abscisses. }\end{array}$ & $\begin{array}{l}\text { Points correspondants } \\
\text { de } \boldsymbol{X}^{\prime \prime} .\end{array}$ & $\begin{array}{l}\text { Valeurs des ordon- } \\
\text { nées de } X^{\prime} \text {. }\end{array}$ & Points singuliers de $\boldsymbol{X}^{\prime}$. \\
\hline$-11,50$ & racine & $\ldots \ldots-6.763 .229$. & \\
\hline$-9,12$ & point de maximum &.-4.4 & p \\
\hline+1 & racine & $\ldots \quad 0,0$ & $\begin{array}{c}\ldots \text { point de maximum et } \\
\text { racine double, }\end{array}$ \\
\hline $\begin{array}{l}+\quad 1,43 \\
+\quad 2,30\end{array}$ & $\begin{array}{l}\text {. point de } r \\
\ldots \quad \text { raci }\end{array}$ & $\begin{array}{l}-20 \\
-115\end{array}$ & $\begin{array}{l}\ldots \text { point d'inflexion, } \\
\ldots \text { point de minimum. }\end{array}$ \\
\hline
\end{tabular}

Pour déterminer les points singuliers de l'équation $\boldsymbol{X}^{\prime}$ qui est du $6^{\text {me }}$ degré, nous aurons donc les valeurs suivantes:

L'équation $\boldsymbol{X}^{\prime}=0$ étant du sixième degré devrait avoir deux points de maximum et trois points de minimum, mais nous voyons d'après le tableau ci dessus, qu'il n'y a que deux points de minimum et un seul de maximum, parconséquent, l'un de ces points est un point composé, et correspond à un couple de racines imaginaires dont nous ne nous occuperons point. - En outre, l'ordonnée du point de maximum étant zéro, cela indique que l'équation a en ce point deux racines égales à +1 . - Des deux racines réelles qu'il nous resie à déterminer, l'un est formée par la branche ascendante gauche, et parconséquent négative et plus grande que l'abscisse $-11,50 \mathrm{du}$ point de minimum situé du côlé négatif; - l'autre racine est formée par la branche ascendante droite, et parconséquent positive et plus grande que l'abssisse $+2,30$, du point de minimum situé du côlé positif. 
Pour déterminer la première de ces racines, substituons successivement dans $X^{\prime}$ les nombres entiers négatifs plus grands que $-11,5$, nous trouverons:

$$
X_{(-14)}^{\prime}=-1.834 .312, \quad X_{(-15)}^{\prime}=+4.685 .520,
$$

ce qui prouve que la racine est entre -14 et -15 ; - mettant aussi -15 dans $\boldsymbol{X}^{\prime \prime}$ nous aurons: $X_{(-15)}^{\prime \prime}=-10.827 .136$, d'où l'on tire les deux nouvelles limites :

et

$$
x=-15-\frac{X_{(-15)}^{\prime}}{X_{(-15)}^{\prime \prime}}=-15+\frac{4.685 .520}{10.827 .136}=-14,56 \ldots
$$

$$
x=-14-\frac{X_{(-14)}^{\prime}}{X_{(-15)}^{\prime \prime}}=-14-\frac{1.834 .312}{10.827 .136}=-14,16 \ldots,
$$

dont la moyenne, avec une seule décimale est: $-14,3 \ldots$ que nous porterons au tableau.

Pour la dernière racine, en substituant les nombres entiers plus grands que $+2,3$, nous trouvons: $X_{(+3)}^{\prime}=+967$, mais puisque $X_{(+2,3)}^{\prime}=-115$, il ' s'ensuit que la racine est entre $+2,3$ et +3 . - En mettant +3 dans $\boldsymbol{X}^{\prime \prime}$ on aura: $\boldsymbol{X}_{(+3)}^{\prime \prime}=+3104$, d'où l'on tire les deux nouvelles limites:

$$
x=+3-\frac{X_{(+3)}^{\prime}}{X_{(+3)}^{\prime \prime}}=+3-\frac{967}{3104}=+2,68 \ldots
$$

et

$$
x=+2,3-\frac{X_{(+2,3)}^{\prime}}{X_{(+3)}^{\prime \prime}}=+2,3+\frac{115}{3104}=+2,33 \ldots .
$$

\begin{tabular}{|c|c|c|c|}
\hline $\begin{array}{l}\text { Valeurs } \\
\text { des } \\
\text { abscisses. }\end{array}$ & $\begin{array}{c}\text { Points correspondants } \\
\text { de } \boldsymbol{X}^{\prime} \text {. }\end{array}$ & $\begin{array}{l}\text { Valeurs des ordon- } \\
\text { nées de } \boldsymbol{X} \text {. }\end{array}$ & Points singuliers de $\boldsymbol{X}$. \\
\hline$-14,3$. & racine & $\ldots+37.645 .498$. & ... point de maximum, \\
\hline$-11,5$ & point de minimum . &.+23.979 .187 . & ... point d'inflexion simple, \\
\hline+1 & $\begin{array}{l}\text { point de maximum } \\
\text { et racine double }\end{array}$ & $\ldots 0$ & $\begin{array}{r}\text { point d'inflexion com- } \\
\text { posé, dont l'axe est la } \\
\text { tangente, et racine triple, }\end{array}$ \\
\hline$+2,3$ & point de & $-69,7$ & ... point d'inflexion simple, \\
\hline$+2,5$ & racine & $-307,82$ & point de minimum. \\
\hline
\end{tabular}

dont la moyenne est $+2,5$ que nous porterons au tableau.

Passons enfin à la recherche des racines de la proposée $\boldsymbol{X}$ pour le lieu géométrique de laquelle nous avons les données suivantes: 
Nous voyons d'après ce tableau que le lieu géométrique de $\boldsymbol{X}$ n'a qu'un seul point de maximum dont l'ordonnée est positive, un seul point de minimum dont l'ordonnée est négative, deux points d'inflexion simples et enfin un point d'inflexion composé dont la tangente est l'axe 'des abscisses, et qui donne trois racines égales à $+1 ;-$ il suit delà, qu'outre les trois racines égales, la courbe a encore deux racines réelles, l'une n'égative et plus grande que $-14,3$ et l'autre positive et plus grande que +2.5 , formées par l'intersection de l'axe avec les branches descendante et ascendante de la courbe. Enfin, deux dernières racines, qui sont imaginaires.

Puisque les trois racines égales sont connues, il ne reste plus qu'à déterminer les deux racines extrêmes; - pour avoir la première, mettons successivement pour $x$ dans l'équation donnée les nombres entiers négatifs plus grands que $-14,3$, qui sont $-15,-16,-17$, etc. nous trouverons:

$$
\boldsymbol{X}_{(-16)}=+24.221 .090 \text { et } \boldsymbol{X}_{(-17)}=-3.780 .800 \text {, }
$$

le changement de signe en passant du nombre -16 au nombre -17 , fait voir que la racine cherchée est entre ces deux nombres.

Comme le nombre -17 correspond à la convexité de la courbe, c'est ce nombre qui doit être près pour bâse d'une nouvelle approximation, nous aurons donc en metlant ce nombre pour $x$ dans la première dérivée:

$$
\boldsymbol{X}_{(-17)}^{\prime}=+38.959 .704 \ldots \text {, }
$$

d'où l'on tire pour deux nouvelles limites:

$$
\begin{aligned}
& x=-17-\frac{X_{(-17)}}{X_{(-17)}^{\prime}}=-17+\frac{3.790 .840}{38.959 .704}=-16,902.953 \ldots, \\
& x=-17-\frac{X_{(-16)}}{X_{(-17)}}=-17-\frac{24.221 .090}{38.959 .704}=-16,621.695 \ldots
\end{aligned}
$$

En substituant ces deux nombres avec une seule décimale, dans $\boldsymbol{X}$, et le plus grand des deux seulement dans la dérivée $\boldsymbol{X}^{\prime}$, il vient:

$$
\begin{aligned}
& \boldsymbol{X}_{(-16,9)}=-18.433,954.769 .9 \ldots, \\
& \boldsymbol{X}_{(-16,6)}=+9.893 .789,316.998 \ldots . \\
& \boldsymbol{X}_{(-16,9)}^{\prime}=+36.504 .369,838.907 \ldots \ldots
\end{aligned}
$$

et pour les deux nouvelles limites:

$$
\begin{aligned}
& x=-16,9-\frac{X_{(-16,9)}}{X_{(-16,9)}^{\prime}}=-16,899.495 .0 \ldots, \\
& x=-16,6-\frac{X_{(-16,6)}}{X_{(-16,9)}^{\prime}}=-16,871.030 \ldots,
\end{aligned}
$$


la racine cherchée est donc comprise entre les deux nombres:

$$
-16,899.495 .0 \ldots \text { et }-16,871.030 \ldots
$$

et comme ces deux nombres n'ont de commun que les nombres entiers et le premier chiffre décimal, 8 , nous ne pouvons considérer que ce seul chiffre comme appartenant à la racine.

Pour former deux nouvelles limites au moyen des deux nombres trouvés, il faudrait prendre ces deux nombres avec toutes leurs décimales, ou au moins avec un assez grand nombre, et les substituer dans $\boldsymbol{X}$ et $\boldsymbol{X}^{\prime}$; mais comme l'opération serait trop longue et trop penible, si l'on conservait un trop grand nombre de chiffres décimaux, il est nécessaire de s'assurer préalablement du nombre de chiffres que l'on doit conserver, et de celui qu'on peut négliger, sans sortir des conditions de l'opération, c'est à dire sans que l'ordonnée change de signe pour la plus grande des deux limites. - En effet, pour la plus petite des deux limites, quel que soit le nombre des chiffres décimaux qu'on néglige, puisqu'on ne fait par là que diminuer cette quantité, on est sûr que cette limite reste moindre que la racine cherchée; - au contraire pour la limite supérieure, si l'on venait à négliger un trop grand nombre de caractères décimaux, il pourrait fort bien arriver qu'on diminuerait trop cette limite, et qu'on la rendrait inférieure à la racine cherchée, de supérieure qu'elle était. -

Pour connaître le nombre des chiffres à conserver, il faut employer l'équation :

$$
i^{\prime}=-i^{2} \cdot \frac{X_{(\alpha)}^{\prime \prime}}{X_{(\alpha)}^{\prime}}
$$

que nous avons déduite dans l'article précédent; nous avons ici $\alpha=-16,9$, $\alpha^{\prime}=-16,899.495 .0$, d'où l'on tire:

$$
\alpha-\alpha^{\prime}=i=0,000.519 \ldots, \text { ou simplement : } i=0,0005 \ldots
$$

de plus en mettant $-16,9$ dans la dérivée du second ordre $\boldsymbol{X}^{\prime \prime}$, on aura:

$$
X_{(-16,9)}^{\prime \prime}=-24.047 .479,7 \ldots \ldots \text {, }
$$

et comme:

$$
X_{(-16,9)}^{\prime}=+36.504 .369,8 \ldots .
$$

on en tire:

$$
\frac{X_{(-16,9)}^{\prime}}{X_{(-16,9)}^{\prime}}=-\frac{24.047 .479,7 \ldots}{36.504 .369,8 \ldots}=-0,658 \text {, }
$$


d'où :

$$
i^{\prime}=-i^{2} \frac{X_{(\alpha)}^{\prime \prime}}{X_{(\alpha)}^{\prime}}=-(0,0005)^{2} \cdot(-0,658)=+0,000.000 .164 .50 \ldots
$$

(D'après la position de la courbe il est évident que la racine cherchée et l'accroissement $i^{\prime}$ sont de signes contraires.) Ce résultat prouve que l'accroissement $i^{\prime}$ d'une nouvelle opération, ne pourrait affecter que la $7^{\text {me }}$ décimale du résultat précédent, et la sixième si la septième est moindre que l'unité; or, dans le cas actuel nous avons $-16,899.495 .0 \ldots$ dont le $7^{\text {me }}$ chiffre décimal est zéro, d'où il suit qu'en retranchant de ce nombre la valeur trouvée pour $i^{\prime}$, la sixime décimale sera aussi affectée et l'on aurait $-16,899.494 \ldots$. valeur approchée, dont les cinq premiers chiffres décimaux sont les mêmes que dans le nombre précédent et parconséquent exacts et appartiennent à la racine; - on voit donc, que pour trouver une nouvelle limite supérieure a la racine, et plus approchée que la précédente, il faut conserver les six premiers chiffres décimaux de cette dernière.

Il semblerait d'après ce résultat que la méthode des deux limites se trouve en défaut, puisqu'elle n'indique qu'un seul chiffre décimal certain, tandis que nous venons de voir qu'il $y$ en a cinq, et cependant, nous eussions pu prévoir d'avance ce résultat, en comparant les valeurs trouvées pour $\boldsymbol{X}_{(-16,9)}$ et $\boldsymbol{X}_{(-16,6)}$, car la seconde valeur est plus de 500 fois plus grande que la première, ou si l'on veut, l'ordonnée correspondante à l'abscisse $(-16,6)$ est plus de cinq cent fois plus grande que celle qui correspond à l'abscisse $(-16,9)$, d'où il résulte clairement que la racine est beaucoup plus proche de ce dernier nombre. - D'ailleurs, en poursuivant par la méthode des deux limites, on arriverait, quoique moins vîte, au même résultat; - en effet, si nous prenons pour bâse d'une nouvelle opération les nombres trouvés pour les deux dernières limites, en conservant seulement les deux premières décimales, nous aurons pour la limite supérieure le nombre $-16,89$ qui étant substitué dans $\boldsymbol{X}$, donne:

$$
\boldsymbol{X}_{(-16,89)}=+345.408,732.335 \ldots
$$

Mais, puisque le résultat de cette substitution a le signe + au lieu du signe qu'il devrait avoir, il s'ensuit que le nombre substitué $(-16,89)$ est plus petit que la racine, et ne peut pas servir de limite supérieure, mais aussi comme il est beaucoup plus approché de la racine que la limite inférieure précédente $(-16,6)$, ainsi en prenant $-16,89$ an lieu de $-16,6$, nous aurons pour 
limite inférieure :

$$
x=-16,89-\frac{X_{(-16,89)}}{X_{(-16,9)}^{\prime}}=-16,89-\frac{345.408 \ldots}{36.512 .369 \ldots}=-16,89945 \ldots
$$

Les quatre premiers chiffres décimaux de cette nouvelle limite, étant les mêmes que les quatre premiers chiffres décimaux de la limite supérieure, nous en concluons que ces quatre chiffres sont exacts, et parconséquent pour continuer la recherche des limites, il faudra prendre pour la limite supérieure au moins les cinq premiers chiffres décimaux de la limite supérieure précédemment trouvée; et, si le résultat de la substitution avait encore le signe + , nous déterminerions une nouvelle limite inféreure encore plus approchée, et qui aurait alors cinq chiffres communs avec la limite supérieure; - on continuerait ainsi jusqu’à ce que le nombre des chiffres décimaux conservés soit tel, que le résultat de la substitution ait le signe qui convient à la limite supérieure. Je ne pousserait pas plus loin ce résultat, qui ne diffère de la vraie racine que de moins d'un millionième d'unité, mais il est facile de voir qu'en prenant pour bâse d'une nouvelle approximation le nombre trouvé, avec les six premiers chiffres décimaux, l'opération suivante devra donner au moins dix chiffres exacts, la suivante 20 , et ainsi de suite, mais on fera bien chaque fois, de s'assurer du nombre des chiffres exacts, au moyen de l'équation $i^{\prime}=-i^{2} \frac{X_{(\alpha)}^{\prime \prime}}{X_{(\alpha)}^{\prime}}$ comme nous l'avons fait dans cet exemple.

Il ne nous reste plus pour terminer l'analyse de l'équation donnée, qu'à déterminer la dernière racine réelle, qui est, comme nous l'avons vu, positive et plus grande que $+2,5$. - Or en essayant d'abord le nombre +3 , nous trouvons:

$$
\boldsymbol{X}_{(+3)}=+80
$$

ce qui prouve que la racine est entre $+2,5$ et +3 , puisque l'ordonnée $\boldsymbol{X}_{(+25)}$ est négative; - ainsi en mettant aussi +3 dans $\boldsymbol{X}^{\prime}$ nous aurons:

$$
\boldsymbol{X}_{(+3)}^{\prime}=+984 \text {, }
$$

d'où nous aurons une première approximation:

$$
x=+3-\frac{X_{(+3)}}{X_{(+3)}^{\prime}}=+3-\frac{80}{984}=+2,918.699 .1 \ldots
$$

Pour une seconde approximation, prenons $+2,9$ en négligeant le reste des 
chiffres décimaux, nous aurons:

$$
\boldsymbol{X}_{(+2,9)}=+0,232.099 .9 \ldots \text {, }
$$

résultat qui fait voir que l'ordonnée de l'abscisse $+2,9$ est fort petite, et que le nombre $+2,9$ est parconséquent très près de la racine; - mettant donc aussi $+2,9$ dans les deux dérivées: $\boldsymbol{X}^{\prime}$ et $\boldsymbol{X}^{\prime \prime}$ nous aurons:

$$
\begin{aligned}
& \boldsymbol{X}_{(+2,9)}^{\prime}=+826,922707 \ldots, \\
& \boldsymbol{X}_{(+2,9)}^{\prime \prime}=+3073,948 \ldots
\end{aligned}
$$

Au moyen de la première de ces valeurs nous trouvons pour seconde approximation :

$$
x=+2,9-\frac{X_{(+2,9)}}{X_{(+2,9)}^{\prime}}=+2,899.719 .32 \ldots
$$

- Pour connaître le nombre de chiffres décimaux exacts de cette valeur, nous aurons

$$
i=+2,9-2,899 \cdot 719.32=+0,00028 \ldots \text { en ne gardant que } 5 \text { décimales, }
$$
d'où,

$$
i^{\prime}=-i^{2} \frac{X_{(+2,9)}^{\prime \prime}}{X_{(+2,9)}^{\prime}}=-(0,00028)^{2}\left\{\frac{3073,848}{826,922707}\right\}=-0,000000292 \ldots,
$$

et, puisque le premier chiffre significatif de cette valeur se trouve au $7^{\text {me }}$ rang des décimales, et moindre que la $7^{\text {ine }}$ décimale du résultat précédent, il sensuit, qu'une nouvelle approximation ne saurait affecter le résultat précédent qu'à partir de la $7^{\mathrm{me}}$ décimale et parconséquent que les six premiers chiffres décimaux déjà trouvés sont exacts et appartiennent à la racine cherchée. - Il faudra donc pour une nouvelle opération conserver les sept premiers caractères décimaux de la valeur précédente, et l'on obtiendrait au moins douze chiffres décimaux exacts.

Je ne pousserai pas plus loin cette recherche, ce que j'ai dit me paraissant suffisant pour l'intelligence de la méthode, mais dans l'article suivant je vais indiquer l'application qu'on en peut faire, à l'extraction des racines des nombres, quel que soit l'exposant du radical. 


\section{XI.}

Soit $\boldsymbol{A}$ un nombre quelconque dont on demande la racine $\boldsymbol{m}^{\text {ième }}$; on aura, en nommant $x$ la racine cherchée:

$$
\boldsymbol{x}=\stackrel{m}{\sqrt{A}},
$$

ou bien en élevant à la $m^{\text {ième }}$ puissance:

$$
\boldsymbol{x}^{m}-\boldsymbol{A}=\mathbf{0},
$$

équation que nous pouvons résoudre par la méthode exposée dans les articles précédents.

Supposons par exemples, que le nombre donné $\boldsymbol{A}$ soit égal à 6589, dont on demande la racine $3^{\text {ine }}$, on aura: $m=3$, et

$$
\boldsymbol{X}=+x^{3}-6589=0,
$$

dont les dérivées sont:

$$
\begin{aligned}
& \boldsymbol{X}^{\prime}=+3 x^{2}, \\
& \boldsymbol{X}^{\prime \prime}=+6 x, \\
& \boldsymbol{X}^{\prime \prime \prime}=+6 .
\end{aligned}
$$

Nous voyons d'après ces équations, que le lieu géométrique de la première n'a qu'un seul point où la touchante soit parallèle à l'axe; ce point correspond à $x=0$, et $\boldsymbol{X}_{(\mathrm{I})}=-6589$; - comme $x=0$ donne aussi $\boldsymbol{X}_{(\mathrm{II})}^{\prime \prime}=\mathbf{0}$ on en conclut que ce point est un point d'inflexion, qui correspond à deux racines imaginaires, et dont partent les deux branches de la courbe, l'une descendente, du côté des $x$ négatifs, l'autre ascendante du côté des $x$ positifs, et la racine réelle de l'équation sera formée par l'intersection de l'axe avec la branche ascendante, et sera un nombre positif; - on voit avec un peu d'attention que ce nombre doit être composé de deux chiffres, parconséquent, en mettant dans $X$ les nombres de deux chiffres, $+10,+20,+30$ etc. on arrivera à deux résultals de signes différents, ainsi on trouvera:

$$
\boldsymbol{X}_{(+1))}=-5589 \text { et } \boldsymbol{X}_{(+2))}=+1411,
$$

ce qui prouve que la racine cherchée est entre +10 et +20 . - En prenant la moyenne de ces deux nombres qui est +15 , nous aurons $\boldsymbol{X}_{(+15)}=-3214$, ce qui prouve que la racine est entre +15 et +20 , et plus près de ce dernier nombre, nous mettrons donc successivement +18 , et +19 , et nous trouverons $\boldsymbol{X}_{(+18)}=-757$ et $\boldsymbol{X}_{(+19)}=+270$, ce qui prouve que la racine 
est entre les deux nombres entiers +18 et +19 , qui ne diffèrent entr'eux que de l'unité.

L'abscisse +19 étant celle qui correspond à la convexité de la courbe. nous la prendrons pour bâse de l'approximation, et nous aurons en la substituant dans $\boldsymbol{X}^{\prime}$,

$$
\boldsymbol{X}_{(+19)}^{\prime}=+1083
$$

d'où

$$
x=+19-\frac{270}{1083}=+18,750792 \ldots \text { pour première valeur approchée. }
$$

En prenant pour seconde valeur approchée, $+18,75$ on aura:

$$
\boldsymbol{X}_{(+18,75)}=+2,796.875 \text { et } \boldsymbol{X}_{(+18,75)}^{\prime}=+1054,687500 \ldots \text {, }
$$

d'où l'on tire la seconde approximation:

$$
x=+18,75-\frac{2,796875}{1054,687500}=+18,747.348 .14 \ldots
$$

Pour connaître le nombre de chiffres exacts que donne cette dernière approximation, posons $i=\boldsymbol{X}_{(+19)}-\boldsymbol{X}_{(+18,75)}=+0,00265 \ldots$ et substituons : $+18,75$ dans $X^{\prime \prime}$, nous aurons: $X_{(+18,75)}^{\prime \prime}=+112,50$, d'où l'on tire:

$$
\frac{\dot{X}_{(+18,75)}^{\prime \prime}}{X_{(+18,75)}^{\prime}}=\frac{+112,5000}{+1054,6875}=0,10666 \ldots \text {, }
$$

parconséquent l'équation en $i^{\prime}$ et $i$, sera:

$i^{\prime}=-i^{2} \frac{X_{(+18,75)}^{\prime \prime}}{X_{(18,75)}^{\prime}}=-(0,00265)^{2}(0,10666 \ldots)=0,000.000 .748 .598 .50 \ldots$, mais comme le $7^{\text {ine }}$ chiffre décimal de cette valeur, surpasse le $7^{\text {me }}$ chiffre décimal de $\boldsymbol{X}_{(+18,75)}$ il s'ensuit que quand on fera une nouvelle approximation, le $6^{\text {the }}$ chiffre seulement du résultat précédent se trouverait affecté; - ainsi, la dernière approximation a cinq chiffres décimaux exacts, et ne diffère de la racine que de moins d'un cent-millième d'unité.

En prenant le nombre trouvé, avec les six premières décimales pour bàse d'une nouvelle approximation, on aura:

$$
\begin{aligned}
& \boldsymbol{X}_{(+18,747.348)}=+0,000.239 .343 .448 .208 .192 \ldots \text { en nous arrêtant à } 18 \text { dé- } \\
& \boldsymbol{X}_{(+18,747.348)}^{\prime}=+1054,389.171 .099 .312 .000 .000 \ldots, \\
& \boldsymbol{X}_{(+18,747.348)}^{\prime \prime}=+112,484.088 .000 .000 .000 .000 \ldots,
\end{aligned}
$$


d'où la nouvelle approximation sera donnée par:

et en effectuant le calcul:

$$
x=+18,747.348-\frac{X_{(+18,747.348)}}{X_{(+18,747.348)}^{\prime}}
$$

$x=+18,747.347 .773 .002 .744 .349 \ldots$ avec dix-huit décimales.

Pour savoir le nombre des chiffres exacts, nommerons $i$ la différence entre cette valeur et la précédente, qui est: $i=0,000.000 .22 \ldots$ en ne conservant que les deux premiers chiffres significatifs, ce qui suffit, d'où l'équation en $i$ et $i^{\prime}$ donne:

ou, en effectuant les calculs :

$$
i^{\prime}=-(0,000.000 .22)^{2} \cdot \frac{X_{(+18,747.348)}^{\prime \prime}}{X_{(+18,747.348)}^{\prime}}
$$

$$
i^{\prime}=-0,000.000 .000 .000 .005 .130 .4 \ldots
$$

Comme le $15^{\text {me }}$ chiffre décimal de cette valeur est plus fort que la quinzième décimale du résultat précédent, il s'ensuit qu'une nouvelle opération n'affecterait le nombre trouvé qu'à partir du $14^{\text {me }}$ chiffre décimal, et que parconséquent les 13 premières décimales sont exactes et appartiennent à la racine cherchée, on aura donc:

$$
x=+18,747.347 .773 .002 .7
$$

pour la racine cherchée, avec 13 chiffres décimaux ce qui est bien suffisant dans la plupart des calculs, à moins qu'on ne veuille pousser l'approximation plus loin pour reconnaître si la partie décimale ne forme pas une fraction périodique.

On voit aisément que la marche du calcul eût été exactement semblable pour toute autre racine que celle du $3^{\text {me }}$ degré, et nous ne nous arrêterons pas davantage sur cette application, nous observerons seulement que le problême général de l'extraction des racines doit se diviser en quatre cas distincts, dont chacun donne une position particulière au lieu géométrique, ainsi: mn étant un nombre entier quelconque, nous aurons:

10. $x=\sqrt{2 m+1} \boldsymbol{A}$ ou $x^{2 m+1}-A=0$, pour la racine d'un degré impair du nombre positif $+\boldsymbol{A} ;-$ cette racine est formé par l'intersection de l'axe avec la branche ascendante; - voir la (fig. 14).

$2^{\circ} . \quad x=V^{2 m+1} A$ ou $x^{2 m+1}+A=0$, pour la racine impaire du nombre négritif - $\boldsymbol{A}$; - cette racine est formée par l'intersection de l'axe avec la branche descendante de la courbe; - voir la (fig. 15). 
$3^{\circ} . x= \pm V^{2 m}+A$ ou $x^{2 m}-A=0$, pour la racine d'un degré pair du nombre positif $+\boldsymbol{A}$; - les deux racines égales et de signes contraires sont formées par les intersections de l'axe avec les deux branches de la courbe, qui sont toutes deux ascendantes; - voir la (fig. 16).

$4^{\circ}$. Enfin $x= \pm \gamma^{2 m}-\boldsymbol{A}$ ou $x^{2 m}+\boldsymbol{A}=\mathbf{0}$, pour la racine d'un degré pair du nombre négatif - A; - la courbe est située comme on le voit dans la (fig. 17) et ne coupe l'axe en aucun point.

Saratoff 1853. 